\title{
A crowd of emotional voices influences the perception of emotional faces: Using adaptation, stimulus salience, and attention to probe audio-visual interactions for emotional stimuli
}

\author{
Sarah C. Izen ${ }^{1} \cdot$ Vivian M. Ciaramitaro ${ }^{1}$ \\ Published online: 15 September 2020 \\ (C) The Psychonomic Society, Inc. 2020
}

\begin{abstract}
Correctly assessing the emotional state of others is a crucial part of social interaction. While facial expressions provide much information, faces are often not viewed in isolation, but occur with concurrent sounds, usually voices, which also provide information about the emotion being portrayed. Many studies have examined the crossmodal processing of faces and sounds, but results have been mixed, with different paradigms yielding different results. Using a psychophysical adaptation paradigm, we carried out a series of four experiments to determine whether there is a perceptual advantage when faces and voices match in emotion (congruent), versus when they do not match (incongruent). We presented a single face and a crowd of voices, a crowd of faces and a crowd of voices, a single face of reduced salience and a crowd of voices, and tested this last condition with and without attention directed to the emotion in the face. While we observed aftereffects in the hypothesized direction (adaptation to faces conveying positive emotion yielded negative, contrastive, perceptual aftereffects), we only found a congruent advantage (stronger adaptation effects) when faces were attended and of reduced salience, in line with the theory of inverse effectiveness.
\end{abstract}

Keywords Multisensory emotional processing $\cdot$ Principle of inverse effectiveness $\cdot$ Visual salience $\cdot$ Crossmodal

The expression of emotions is inherently multimodal (Robins, Hunyadi, \& Schultz, 2009), with emotional information conveyed via faces, voices, odors, touch, and body posture or movement (Bestelmeyer, Maurage, Rouger, Latinus, \& Belin, 2014; Herz, McCall, \& Cahill, 1999; Niedenthal,

Public significance statement

This study highlights how the processing of emotional information in a face is influenced by concurrent emotional information in a different sense. We found that faces and sounds of matched emotional valence yield a perceptual benefit compared with faces and sounds that do not match, but only when faces are reduced in salience, and when the emotion of the face is attended. This extends basic principles of multisensory interactions, the principles of inverse effectiveness, to static emotional stimuli and highlights the importance of considering stimulus salience and attention in the study of emotional processing across the senses.

Electronic supplementary material The online version of this article (https://doi.org/10.3758/s13414-020-02104-0) contains supplementary material, which is available to authorized users.

Vivian M. Ciaramitaro

Vivian.ciaramitaro@umb.edu

1 Department of Psychology, Developmental and Brain Sciences, University of Massachusetts Boston, 100 Morrissey Boulevard, McCormack, M-04-212, Boston, MA 02125, USA
2007; Zald \& Pardo, 1997). Our ability to extract a person's emotional state, identify the potential threat they pose, and act accordingly is crucial to social interaction. By combining auditory and visual information, we should be able to optimize emotional processing.

From very early in life, we know what visual and auditory emotional information goes together. Infants as young as 6.5 months of age can match the emotions conveyed by a face with the corresponding emotions conveyed by a voice (Grossmann, Striano, \& Friederici, 2006; Vogel, Monesson, \& Scott, 2012). The combining of information across multiple senses has been shown to be beneficial to decoding others' emotional state. For example, infants as young as 4 months of age can discriminate emotions when presented with bimodal (visual and auditory) stimuli, whereas discrimination of unimodal (either auditory or visual) stimuli alone does not occur until later in development, at 5 and 7 months, respectively (Flom \& Bahrick, 2007). What is less clear is how emotional information in one sensory modality can influence our perception of emotional information in another sensory modality.

One powerful tool used to reveal the space in which faces are represented is adaptation (see Webster \& MacLeod, 2011, for a review). Repeated stimulus exposure downregulates neuronal firing in response to that stimulus, resulting in a 
perceptual change, or contrastive aftereffect. For example, repeated exposure to female faces can bias androgynous faces to appear more masculine (Little, Feinberg, DeBruine, \& Jones, 2013). Many features of a face can be adapted, such as gender, ethnicity, and even emotions (Little et al., 2013; Rutherford, Chattha, \& Krysko, 2008; Webster, Kaping, Mizokami, \& Duhamel, 2004) as well as combinations of face features (i.e., contingent adaptation between gender and ethnicity; $\mathrm{Ng}$, Ciaramitaro, Anstis, Boynton, \& Fine, 2006) or between gender and emotion (Harris \& Ciaramitaro, 2016).

In the visual domain, adapting to negative emotional faces can shift emotional perception to be more positive, biasing neutral faces to appear happier (e.g., Rutherford et al., 2008), whereas adapting to positive emotional faces can shift emotional perception to be more negative, biasing neutral faces to appear sadder (e.g., Rutherford et al., 2008), angrier (Webster et al., 2004), or more fearful (Hsu \& Young, 2004). Similarly, in the auditory domain, adapting to negative emotional sounds can shift emotional perception to be more positive, biasing neutral voices to appear happier and vice versa (P. E. G. Bestelmeyer, Rouger, DeBruine, \& Belin, 2010). It has also been shown that the representation of emotion is supramodal, such that adapting to emotional information in one modality can alter perceptual biases in another modality. For example, adapting to visual emotions yields contrastive aftereffects for auditory emotions (Pye \& Bestelmeyer, 2015; Skuk \& Schweinberger, 2013), and adapting to auditory emotions yields contrastive aftereffects for visual emotions (Wang et al., 2016).

Given evidence for emotional adaptation within and across the senses, what is the influence of having emotional content presented concurrently in different senses, as is often the case in our everyday experience? Few studies have considered the perceptual consequences for concurrent auditory and visual emotions and results have been equivocal. For example, de Gelder and Vroomen (2000) found that an emotional voice, happy or sad, could bias perception of a simultaneously presented neutral face to match that of the voice. Similarly, Müller et al. (2011) found that emotional sounds (e.g., screams) could bias perception of a simultaneously presented neutral face to appear more fearful compared with presentation of neutral sounds (e.g., yawns). Chen et al. (2016), using a change-detection paradigm, found increased accuracy and reduced reaction times for detecting changes involving bimodal compared with unimodal stimuli (Chen et al., 2016). However, using adaptation paradigms, Fox and Barton (2007) found no biased perception of emotional faces from emotional sounds, and Wang et al. (2016) found no benefit (no increased adaptation) when bimodal (visual and auditory) stimuli were of the same positive emotional valence (congruent) compared with a unimodal visual stimulus, suggesting that auditory emotional information carried little weight in biasing face perception. In a previous study, using an adaptation paradigm similar to the one used here for Experiment 1, but using negative facial emotions, we also found no benefit when visual and auditory stimuli were congruent (angry faces and negative sounds), compared with a unimodal visual condition (angry faces) or an incongruent condition (angry faces and positive sounds; Izen, Lapp, Harris, Hunter, \& Ciaramitaro, 2019).

Discrepant results across adaptation studies may arise from differences in experimental paradigms. For instance, many adaptation paradigms use only a few exemplar faces and/or voices, especially since aftereffects are stronger after adaptation to the same versus different faces (Fox \& Barton, 2007). However, to test for a supramodal representation and for interactions between visual and auditory emotional stimuli, providing many exemplars helps assure one is not adapting to the unique configuration of features of a given face or voice rather than to emotion, per se. Furthermore, if only a few faces and voices are used and presented as unique pairs, presentation of a stimulus in one modality might induce imagery of the associated stimulus in the other modality. In this case, imagery, not crossmodal interactions, would drive the transfer of adaptation effects across modalities. To promote adaptation to emotion rather than to unique configurations of features of a given face, and to prevent induced imagery from learned associations of unique face-voice pairs, the current study used 30 unique faces and 15 unique crowd sounds selected at random during adaptation.

As an additional means to ensure participants are adapted to emotion and not to features of particular identities or to unique face-voice pairs, the current study used crowd sounds (voices from a group of individuals) rather than voices from a single individual. In the visual domain, crowds of faces have been shown to be more informative than single faces. For example, the gaze of a group can be more effective than the gaze of an individual in directing attention (Gallup et al., 2012). Given that the mean emotion of a group of faces can be extracted without representing individual characteristics (Haberman, Harp, \& Whitney, 2009; Haberman \& Whitney, 2007), we expected the mean emotion of a group of voices could also be extracted without representing individual voices.

We used the strength of adaptation as a proxy for the strength of interactions between visual and auditory emotional information. Adaptation was quantified by fitting data with a psychometric function and calculating changes in the point of subjective equality (PSE) at baseline versus postadaptation. We also quantified changes in the slope of the psychometric function at baseline versus postadaptation. The slope of the psychometric function can provide information regarding the extent to which stimuli are perceived as categorical as well as provide an estimate of variance in the data (Roesch, Sander, Mumenthaler, Kerzel, \& Scherer, 2010).

We hypothesized that adaption would be stronger to happy faces presented with positive sounds (congruent emotional 
valence), resulting in a larger perceptual aftereffect and steeper slope, compared with adapting to happy faces presented with negative sounds (incongruent emotional valence). Furthermore, we expected the strength of adaptation to faces alone to either fall in between the congruent and incongruent conditions, or to show no difference from the congruent condition, given previous results by Wang et al. (2016) using an adaptation paradigm and happy faces.

\section{Experiment 1}

\section{Method}

Participants A total of 83 participants, 18-34 years of age, were recruited from the University of Massachusetts Boston community. A G*Power analysis (Faul, Erdfelder, Lang, \& Buchner, 2007) for a planned analysis of variance (ANOVA) with one between-subjects factor, using an alpha error of 0.05 , power of .95, and an effect size of 0.5 , estimated a total sample size of roughly 66 participants across the three conditions of our study. Thus, we aimed to collect more participants, assuming some data loss. Nineteen participants were excluded due to the following: experimenter error (7); biased behavioral responses (6), where $80 \%$ happy faces were judged happy less than $75 \%$ of the time or $80 \%$ angry faces were judged happy more than $25 \%$ of the time; or participant error (6), where participants failed to press the correct buttons to make their responses. Our final sample consisted of 64 participants, 49 females (mean age $=21.44$ years; $S D=0.417$; range: $18-29$ years) and 13 males (mean age $=24.08$ years; $S D=1.602$; range: 19-34 years), with two participants not reporting their gender and seven not reporting their age. Please see Table 1 for detailed demographics of our final sample.

Participants reported normal hearing and normal or corrected-to-normal vision, provided written informed consent, and were compensated $\$ 20$ or received extra credit for an eligible undergraduate course. All experimental procedures and protocols were approved by the University of Massachusetts Boston Institutional Review Board and complied with the Declaration of Helsinki.

Apparatus Participants were seated $45 \mathrm{~cm}$ from a Nexus CRT monitor and were positioned on a chin and forehead rest to maintain stable head position and constant viewing distance. Responses were recorded via laptop keyboard button press or Cedrus Response Box using MATLAB and the Psychophysics Toolbox (Brainard, 1997; Kleiner, Brainard, \& Pelli, 2007; Pelli, 1997). In order to minimize distractions from ambient sounds, auditory stimuli were presented via noise-cancelling headphones (3M-Peltor headset).
Table 1 Demographic information (Experiment 1)

\begin{tabular}{|c|c|c|c|c|}
\hline $\begin{array}{l}\text { Demographics } \\
\text { Mean age } \\
(S D)\end{array}$ & & $\begin{array}{l}\text { Congruent } \\
22.21(3.17)\end{array}$ & $\begin{array}{l}\text { Incongruent } \\
21.70 \\
(3.77)\end{array}$ & $\begin{array}{l}\text { Visual } \\
22.11(4.17)\end{array}$ \\
\hline \multicolumn{5}{|l|}{ White } \\
\hline Male & $N$ & $3(60 \%)$ & $2(100 \%)$ & $2(33.3 \%)$ \\
\hline Female & $N$ & $9(50 \%)$ & $6(31.6 \%)$ & $4(33.3 \%)$ \\
\hline \multicolumn{5}{|l|}{ Hispanic } \\
\hline Male & $N$ & $1(20 \%)$ & $0(0 \%)$ & $0(0 \%)$ \\
\hline Female & $N$ & $3(16.7 \%)$ & $7(36.8 \%)$ & $3(25 \%)$ \\
\hline \multicolumn{5}{|c|}{ African/African American } \\
\hline Male & $N$ & $0(0 \%)$ & $0(0 \%)$ & $1(16.7 \%)$ \\
\hline Female & $N$ & $2(11.1 \%)$ & $0(0 \%)$ & $1(8.3 \%)$ \\
\hline \multicolumn{5}{|l|}{ Asian } \\
\hline Male & $N$ & $0(0 \%)$ & $0(0 \%)$ & $2(33.3 \%)$ \\
\hline Female & $N$ & $2(11.1 \%)$ & $5(26.3 \%)$ & $3(25 \%)$ \\
\hline \multicolumn{5}{|l|}{ Multiracial } \\
\hline Male & $N$ & $0(0 \%)$ & $0(0 \%)$ & $0(0 \%)$ \\
\hline Female & $N$ & $0(0 \%)$ & $0(0 \%)$ & $0(0 \%)$ \\
\hline \multicolumn{5}{|l|}{ Unspecified } \\
\hline Male & $N$ & $1(20 \%)$ & $0(0 \%)$ & $1(16.7 \%)$ \\
\hline Female & $N$ & $2(11.1 \%)$ & $1(5.3 \%)$ & $1(8.3 \%)$ \\
\hline
\end{tabular}

Stimuli Fifteen unique male and 15 unique female face images, with at least $75 \%$ validity ratings in judging happy emotions, were chosen from the NimStim Face Stimulus database (Tottenham et al., 2009) to be used as adaptation stimuli. All faces were grayscaled to $50 \%$ and embedded in a gray oval to obscure nonfacial features, such as hair (see sample stimuli in Fig. 1; D. A. Harris \& Ciaramitaro, 2016). All faces were 360 $\times 510$ pixels, subtended $12^{\circ}$ visual angle, and were presented at central fixation. A total of 30 possible faces (21 White, 3 Asian, and 6 Black) could be presented during the adaptation phase of the experiment.

Auditory stimuli were 1-s. clips of crowds expressing positive (such as cheering) or negative (such as booing) emotions. Clips contained no words, to limit the variability in participants' emotional reactions, since words might convey different emotional valences for different participants. Ratings for auditory stimuli were assessed by an independent group of 20 participants who listened to 87 one-second sound clips of positive (39) or negative (48) emotions and judged each on a 6-point Likert scale for ratings of emotional valance (how angry, how happy, and how mocking). Only sound clips with good sound quality and $75 \%$ validity in judgments of happy or angry emotions were used, excluding clips judged as sounding mocking. A total of 15 positive and 15 negative crowd sounds could be presented during the adaptation phase.

For probe faces, a subset of face images was morphed along an emotional continuum using MorphMan software 
Baseline Condition ALL PARTICIPANTS

\section{Adaptation Condition}

\section{ONE CONDITON PER PARTICIPANT}

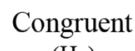

(Hc)

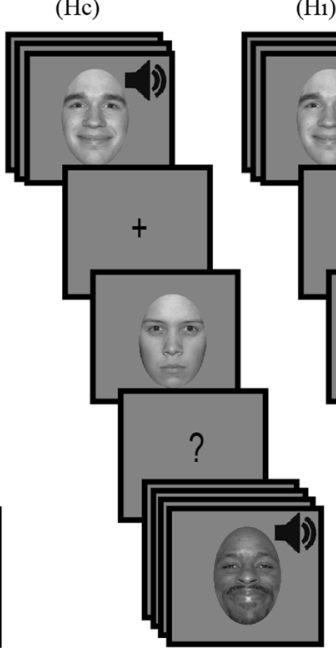

ngruent
Visual Only (Hv)

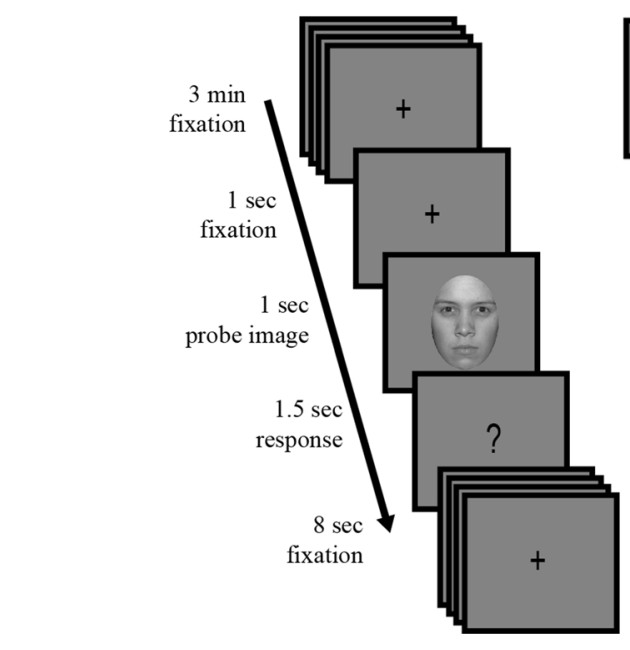

Fig. 1 Experimental procedure (Experiment 1). Participants were presented randomly selected faces that had been morphed along an emotional continuum, from angry to happy (eight unique face identities: four males, four females). They judged each face morph as either happy or angry. For baseline, the block started with a 3-minute fixation, and participants were instructed to maintain central fixation. This was followed by a face morph presented for $1 \mathrm{~s}$, followed by a $1.5-\mathrm{s}$ response period, during which a question mark was presented and participants had to indicate whether they thought the previously presented face morph was happy or angry by pressing a key on a keyboard. Baseline consisted of 64 trials. For adaptation, participants were presented the same face morphs and made the same judgments as during baseline. However, adaptation began with a 3-minute exposure to $100 \%$ happy faces and each face judgment was followed by an 8 -second top-up exposure during which eight $100 \%$ emotional faces were presented, each for $1 \mathrm{~s}$. Adaptation also consisted of 64 trials. A given participant first completed baseline and then completed only one of the three possible adaptation conditions: bimodal adaptation conditions - in which the emotional valence of faces and voices were either matched (happy faces and positive sounds [Hc]) or mismatched (happy faces and negative sounds [Hi]), or a unimodal adaptation condition, in which participants were adapted to happy faces only $(\mathrm{Hv})$
(STOIK Imaging, Moscow, Russia). Eight unique identities, including four female and four males faces, five White, two Asian, and one Black, were morphed from neutral to $10 \%$, $20 \%, 40 \%$, and $80 \%$ happy and the complementary angry morphs. Altogether, there were 72 possible face morph stimuli that could be presented during the test phase.

Procedure The experiment began with three practice trials to familiarize participants with experimental procedure and response timing. Trials started with an auditory alerting cue, followed by a blank oval (1 s), and then a question mark $(1.5 \mathrm{~s})$, during which time participants were instructed to press a button to practice the timing of when to indicate their judgment. Participants were instructed to maintain central fixation throughout the experiment, but eye gaze was not monitored. Following practice trials, each participant completed one baseline and one adapt condition (see Fig. 1), each lasting 15 minutes, with a 5-minute break between.

During baseline, participants viewed a gray screen with a central fixation cross for $180 \mathrm{~s}$. Then, an auditory alerting cue was presented, followed by a probe face selected at random from the 72 possible face morphs $(1 \mathrm{~s})$. Then, a question mark appeared $(1.5 \mathrm{~s})$, and participants judged the face morph as happy or angry via key press. Trials were included for analysis only if participants responded during the 1.5-s response window. Each response was followed by a gray screen with a central fixation cross $(8 \mathrm{~s})$. A total of 64 probe face morphs were presented during baseline. Eight repeats of each of the eight unique face identities were presented for each emotional morph (40\%, 20\%, and 10\% happy or angry and neutral), except at the extremes of the psychometric function, where only four of the eight identities were presented for the $80 \%$ happy and $80 \%$ angry morphs. Responses to probe faces were used to calculate each participant's point of subjective equality (PSE), the face equally likely to be described as happy or angry (described in data analysis).

Adaptation was similar to baseline, but instead of the initial 180 -s fixation cross (initial adaptation), and the 8Postadaptation, the same face morphs as in baseline were presented at random, ands fixation cross between each trial (topup adaptation), $100 \%$ happy faces were presented during initial adaptation and top-up adaptation in one of three possible adaptation conditions: (1) congruent: visual and auditory stimuli matched in emotional valence, such that happy faces were presented concurrently with positive sounds (Hc); (2) incongruent: visual and auditory stimuli mismatched in emotional valence, such that happy faces were presented with negative sounds (Hi); or (3) visual alone: visual stimuli were presented 
in isolation, with no concurrent sound (Hv). These three conditions were run in a between-subjects design, such that a given participant only viewed one of the three possible adaptation conditions.

During initial adaptation, a total of $180,100 \%$ happy faces (30 unique faces) or happy faces and emotional crowd sounds (15 unique emotional sounds) were presented at random (1 s each), avoiding the creation of unique face-sound pairs. Postadaptation, the same face morphs as in baseline were presented at random, and participants had to judge each face morph happy or angry. During top-up adaptation, eight $100 \%$ happy faces and/or emotional crowd sounds were presented at random $(1 \mathrm{~s}$ each). Judgments from a total of 64 morphed probe faces were assessed postadaptation and were used to determine the change in each participant's unique PSE (described in Data Analysis section).

Data analysis All data were analyzed using MATLAB (The MathWorks, Inc., Natick, MA, USA). We used psignifit (http://bootstrap-software.org/psignifit/), which implements the maximum-likelihood method described by Wichmann and Hill (2001), to fit the data for each participant for the baseline and postadapt condition separately. We plotted the emotional morph continuum along the $x$-axis and the percentage of trials the participant responded that a given face morph appeared happy on the $y$-axis, and fit the data to a cumulative normal function. The PSE conveys the percent of emotion in a face required to perceive the face as emotionally neutral: the face judged $50 \%$ of the time happy and $50 \%$ of the time angry. We plotted happy emotions to have positive values (to the right of zero) and angry emotions to have negative values (to the left of zero). As such, a positive baseline PSE indicates more positive affect is required to see a face as neutral (indicating a negative perceptual bias). Conversely, a negative baseline PSE indicates more negative affect is required to see a face as neutral (a positive perceptual bias).

To quantify the strength of perceptual biases postadaptation, we measured how each participant's judgments of the face considered emotionally neutral at baseline changed postadaptation. We subtracted the $y$-value for the PSE at baseline (defined as .5) from the $y$-value for the PSE for the adaptation block. Importantly, such a quantification normalizes for baseline biases and allows quantification of perceptual biases using the linear range of the $y$-axis. To quantify change in slope postadaptation, we measured the slope of the psychometric function at the PSE for baseline and after adaptation, and subtracted the baseline slope from postadaptation slope measurement.

Figure 2 depicts predicted behavioral effects for each adaptation condition using hypothetical data. For example, if the face judged neutral (50\%) at baseline is judged happy on $25 \%$ of trials postadaptation, the shift is a negative bias of $25 \%$ (50$25)$. Such a negative bias is expected following adaptation to positive emotions (see Fig. 2).
Theoretical Predictions

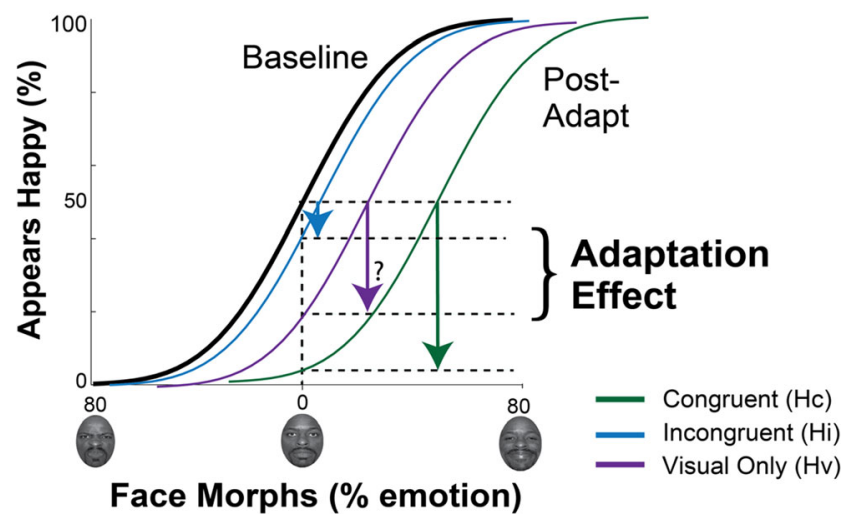

Fig. 2 Behavioral predictions (Experiment 1). The $x$-axis depicts the emotional continuum, from $80 \%$ angry to $80 \%$ happy, with the standard neutral from the NimStim database at zero. The $y$-axis shows the hypothetical percentage of happy judgments. We measured each participant's unique point of subjective equality (PSE): the face the participant judged equally likely to be happy or angry. The thick black curve reflects a cumulative normal fit to judgments of each face morph at baseline. Baseline PSE was quantified as the face morph supporting 50\% happy judgments. The predicted direction and magnitude of changes in the baseline PSE (PSE shift) after adaptation to happy faces are depicted respectively in the direction and magnitude of arrows extending from the baseline PSE. We predicted a negative perceptual bias after adapting to happy faces, a negative PSE shift, with the largest shift for congruent visual and auditory emotional stimuli $(\mathrm{Hc})$, the weakest shift for the incongruent condition (Hi), and a strong to intermediate shift for the visual only (Hv)

We expected shifts or biases in the perception of emotion to vary as a function of adaptation condition. We expected negative bias to be (1) strongest after congruent adaptation (when visual and auditory stimuli both conveyed positive emotional content); (2) weakest after incongruent adaptation (when visual stimuli conveyed positive, but auditory stimuli conveyed negative emotional content); and (3) strong to moderate after adaptation to only visual positive emotional content, with some ambiguity as to whether visual alone would fall between the congruent and incongruent conditions or be similar to the congruent condition. Additionally, we expected changes in slope to vary as a function of adaptation condition. We expected an increase in slope (steeper slope) to be (1) strongest after congruent adaptation, (2) weakest after incongruent adaptation, and (3) strong to moderate after adaptation to only visual positive emotional content.

Statistical analyses For data that were normally distributed, parametric statistics were performed. For data that were not normally distributed, nonparametric statistics were performed, but parametric analyses were also reported for comparison.

Planned statistical analysis included ANOVAs for quantifying both perceptual shifts and changes in slope across adaptation conditions. Because we have an a priori expectation that the perceptual shift will be greater following adaptation to congruent versus incongruent stimuli, and our main interest 
is in the difference between these two conditions, with the visual-only condition serving as a control with less clear outcomes, we also conducted two-tailed $t$ tests to compare perceptual shifts between the congruent and incongruent conditions. In addition, $t$ tests were also used to examine whether perceptual shifts were significantly different from zero, or no change, postadaptation. Finally, we also examined baseline biases that could potentially minimize the size of perceptual or physiological shifts following adaptation.

\section{Results}

Of the 64 total possible trials of face morphs presented to determine perceptual biases in judging emotion, participants completed an average of 58.36 baseline trials $(S D=6.10$; range: $39-64)$ and 60.64 postadaptation trials $(S D=4.62$; range: 41-64). A Kruskal-Wallis ANOVA was run to test the hypothesis that the strength of changes in perception varied across adaptation conditions. We expected the largest perceptual change, shift in PSE, following congruent adaptation, the weakest changes following incongruent adaptation, and a moderate effect following only visual adaptation. We found no significant main effect of adaptation condition on PSE shift, $\chi^{2}(2)=2.09, p=.35$, with a mean rank PSE shift of 34.63 for congruent, 34.59 for incongruent, and 27.11 for

\section{Perceptual Shift}

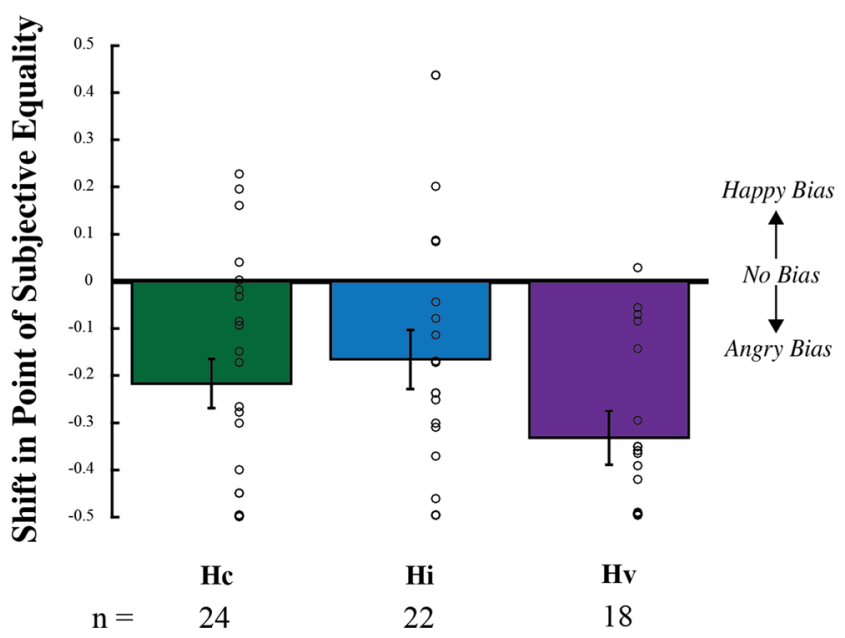

Fig. 3 PSE shifts (Experiment 1). PSE shifts, the change in judgments of the face judged neutral at baseline, are shown for each adaptation condition. The $x$-axis depicts the adaptation condition, and the $y$-axis depicts the mean PSE shift ( $\pm S E M$ across participants), with data from individual participants shown via open circles. A mean shift in the negative direction indicates that the unique face judged neutral at baseline for each participant looked angrier, on average, after adaptation. Conversely, a mean shift in the positive direction indicates that the unique neutral face looked happier, on average, after adaptation. After adaptation to happy faces, we observed a negative shift in all conditions, as expected: $\mathrm{Hc}, t(23)=-3.77$, $p=.001 ; \mathrm{Hv}, t(17)=-6.66, p<.001 ; \mathrm{Hi}, t(21)=-2.72, p=.013$. There were no differences in magnitude of shift between conditions, $\chi^{2}(2)=$ $2.09, p=.35$ visual only (see Fig. 3). Although we found no significant differences in adaptation across conditions, one-sample $t$ tests indicated that all three conditions showed significant adaptation effects (PSE shifts were significantly different from baseline, or zero using a $p$ value threshold of 0.0167 to account for multiple comparisons: Hc, $t(23)=-3.77, p=.001, d=-0.77$; $\mathrm{Hv}, t(17)=-6.66, p<.001, d=-1.56 ; \mathrm{Hi}, t(21)=-2.72, p=$ $.013, d=-0.58$. Thus, adapting to happy faces resulted in neutral faces being judged more negatively, as expected. Furthermore, given our a priori hypothesis that perceptual changes would be greater following congruent versus incongruent stimuli, and because the data were not normally distributed, we ran a Mann-Whitney $U$ test to explicitly compare the congruent and incongruent conditions, but found no significant difference $(p=.98)$.

Despite PSE shifts not differing across adaptation conditions, another quantification of our data, slope, might have differed. Changes in slope are of interest since steeper slopes can be indicative of less variance in perceptual data (i.e., stronger categorical perception; Roesch et al., 2010). Although we found no significant differences in slope changes across adaptation conditions, $F(2,61)=0.47, p=.63$, partial $\eta^{2}=0.02$, for all conditions, the change in slope was positive and significantly different from zero, indicating steeper slopes after adaptation: $\mathrm{Hc}, t(23)=2.71, p=.023, d=0.55$; $\mathrm{Hv}, t(17)=2.46, p=.025, d=.58 ; \mathrm{Hi}, t(21)=4.55, p<.001, d$ $=.97$. Thus, participants viewed the emotional stimuli as more categorical after adaptation. Finally, of note, all our measures quantifying perceptual changes were normalized to baseline. Although baseline biases could have influenced the effects of interest, we found no significant difference in baseline PSE across adapt conditions, $F(2,61)=1.71, p=.18$, partial $\eta^{2}=$ 0.05 .

\section{Experiment 1 discussion}

We assessed the strength of adaptation (i.e., perceptual biases after adaptation) to quantify interactions between concurrent visual and auditory emotional information. Unlike previous work using unique face-voice pairings for only a few individual identities, we used a wide range of face identities and random, unassociated, emotional crowd sounds to assess emotional processing. The rationale for this was to ensure that participants were adapted to the emotional content of the stimuli, rather than to specific features of one or two exemplar faces or voices, decreasing the chances that visual imagery would bias judgments.

We hypothesized that (1) the emotion perceived in a face would show a negative bias postexposure to positive emotional faces, in accord with contrastive perceptual aftereffects, and that (2) such aftereffects would vary based on whether emotion was conveyed by visual or auditory information and whether visual and auditory emotional valence matched. 
Overall, we found exposure to positive emotions yielded negative perceptual biases (negative PSE shift). Contrary to our expectations, the magnitude of PSE shift did not differ between our congruent and incongruent conditions. We also found no difference between the congruent and visual only condition, which accords with previous work using an adaptation paradigm and unique face-voice pairings, comparing congruent happy faces and happy voices to only happy faces without voices (Wang et al., 2016).

One possible explanation for our failure to find differences in adaptation across conditions is that the auditory stimuli (a crowd of sounds conveying multiple individuals) were not associated with the number of visual stimuli (a single individual face). It has been shown that numerosity is modality independent in adults (e.g., Barth, Kanwisher, \& Spelke, 2003), and even infants can match numerosity not only within but across the senses (Jordan \& Brannon, 2006; Kobayashi, Hiraki, \& Hasegawa, 2005; Starkey, Spelke, \& Gelman, 1990). A mismatch in numerosity could have decreased multisensory interactions in Experiment 1, as well as diverted attention to the mismatched sounds. If attention was directed away from the face due to our auditory stimuli, this also could have decreased the strength of adaptation, given that directing attention to emotional information has been shown to enhance the strength of adaptation effects compared with directing attention away from emotional information (e.g., Rhodes et al., 2011).

\section{Experiment 2}

In Experiment 2, we better matched the numerosity of visual and auditory emotional information by presenting a crowd of faces concurrently with the crowd sounds. Previous studies highlight the ease with which the visual system creates an average representation of various elements.

\section{Ensemble perception}

The visual system can quickly and efficiently create average representations for specific visual features, termed ensemble coding. This is not only true for lower level features, such as size, motion, and orientation (Alvarez, 2011; Ariely, 2001; Chong \& Treisman, 2003; Parkes, Lund, Angelucci, Solomon, \& Morgan, 2001), but also for higher level features, such as facial identity (de Fockert \& Wolfenstein, 2009; Haberman et al., 2009; Haberman \& Whitney, 2007). The visual system is capable of quickly extracting the mean identity from a group of faces, more accurately representing a morph of the mean identity than any single identity depicted in the group (de Fockert \& Wolfenstein, 2009). Importantly, with quick and efficient formation of a summary statistic, individual information (such as the size of a visual dot or the expression of an individual face) is lost. Interestingly, it has been shown that information gained from viewing a crowd is better able to guide behavior than information gained from viewing an individual. For instance, participants can extract gaze direction effectively from crowds to aid in orienting to an important stimulus (Sweeny \& Whitney, 2014), and the gaze of a group is more effective at directing attention than is the gaze of an individual (Gallup et al., 2012).

\section{Processing emotional crowds}

The visual system is capable of quickly extracting not only a static visual feature, such as face identity, to determine the mean identity of a group but also a dynamic visual feature, such as face emotion. It has been shown that mean emotional content can be extracted from a crowd of faces, even if no information about an individual face in the group is retained or even if the mean emotion is never displayed by an individual face in the group (Haberman et al., 2009; Haberman \& Whitney, 2007). An example is the "cheerleader effect," where an individual is judged more attractive if amongst an attractive versus an unattractive group of people (Walker \& Vul, 2014). This occurs because of the automaticity of creating ensemble statistics along with the tendency to perceive a single item towards the group average (Brady \& Alvarez, 2011). In this case, the cheerleader effect may cause faces to be perceived as closer to the mean emotion of the group of faces.

Assuming that people process the emotion of a group of faces similarly, or more efficiently, than a single face, how are a concurrent crowd of faces and a crowd of voices processed across the senses? In Experiment 2, we created new face adaptation stimuli consisting of a crowd of faces (a group of four faces arranged in a diamond pattern around central fixation). Importantly, as in Experiment 1, we used multiple voices, a crowd, instead of single voices, to decrease the probability of eliciting visual imagery, since it is unlikely a participant would imagine a single face when hearing a crowd of voices. Additionally, we introduced a questionnaire after baseline and after adaptation to assess changes in the subjective experience of the sounds (how positively or negatively participants experienced the crowd sounds postadaptation relative to baseline).

Given the power of crowds over individuals, we not only expected Experiment 2 to mitigate the problem of mismatched numerosity in Experiment 1 but we also expected to see stronger adaptation effects for visual and auditory stimuli matched in emotional valence (happy faces and positive crowd sounds) compared with unmatched valence (happy faces and negative crowd sounds). We hypothesized that multimodal stimuli better matched in numerosity would yield stronger multisensory interactions and allow us to observe our predicted results: (1) strongest postadaptation biases after congruent adaptation (when visual and auditory stimuli both conveyed similar emotional valence), (3) weakest postadaptation biases after 
incongruent adaptation (when visual and auditory stimuli conveyed different emotional valence), and (3) strong to moderate postadaptation biases after adaptation to only visual emotional content.

\section{Method}

Participants An additional 80 new participants, 18-47 years of age, were recruited from the University of Massachusetts Boston community. We aimed to collect roughly the same number of participants as in Experiment 1. Eleven participants were excluded due to the following: experimenter error (3); biased behavioral responses, as defined for Experiment 1 (3); or insufficient trials completed (5). Our final sample consisted of 69 participants, 49 females (mean age $=23.43$ years; $S D=$ 5.74; range: $18-47$ years), 18 males (mean age $=23.11$ years; $S D=3.51$; range: $18-30$ years), with two participants not reporting their gender or age (please see Table 2 for detailed demographics of our final sample).

Participants reported normal hearing and normal or corrected-to-normal vision, provided written informed consent, and were compensated $\$ 12 / \mathrm{hr}$ or received extra credit for an eligible undergraduate course. All experimental procedures and protocols were approved by the University of Massachusetts Boston Institutional Review Board and complied with the Declaration of Helsinki.

Table 2 Demographic information (Experiment 2)

\begin{tabular}{|c|c|c|c|c|}
\hline $\begin{array}{l}\text { Demographics } \\
\text { Mean age } \\
(S D)\end{array}$ & & $\begin{array}{l}\text { Congruent } \\
25.20 \\
(7.05)\end{array}$ & $\begin{array}{l}\text { Incongruent } \\
20.95 \\
(2.73)\end{array}$ & $\begin{array}{l}\text { Visual } \\
23.65 \\
(3.47)\end{array}$ \\
\hline \multicolumn{5}{|l|}{ White } \\
\hline Male & $N$ & $2(28.6 \%)$ & $3(50 \%)$ & $1(20 \%)$ \\
\hline Female & $N$ & $7(38.9 \%)$ & $5(31.3 \%)$ & $4(26.7 \%)$ \\
\hline \multicolumn{5}{|l|}{ Hispanic } \\
\hline Male & $N$ & $1(14.3 \%)$ & $2(33.3 \%)$ & $1(20 \%)$ \\
\hline Female & $N$ & $2(11.1 \%)$ & $4(25 \%)$ & $1(6.7 \%)$ \\
\hline \multicolumn{5}{|c|}{ African/African American } \\
\hline Male & $N$ & $1(14.3 \%)$ & $1(16.7 \%)$ & $1(20 \%)$ \\
\hline Female & $N$ & $3(16.7 \%)$ & $3(18.8 \%)$ & $0(0 \%)$ \\
\hline \multicolumn{5}{|l|}{ Asian } \\
\hline Male & $N$ & $3(42.9 \%)$ & $0(0 \%)$ & $1(20 \%)$ \\
\hline Female & $N$ & $5(27.8 \%)$ & $4(25 \%)$ & $5(33.3 \%)$ \\
\hline \multicolumn{5}{|l|}{ Multiracial } \\
\hline Male & $N$ & $0(0 \%)$ & $0(0 \%)$ & $0(0 \%)$ \\
\hline Female & $N$ & $1(5.6 \%)$ & $0(0 \%)$ & $1(6.7 \%)$ \\
\hline \multicolumn{5}{|l|}{ Unspecified } \\
\hline Male & $N$ & $0(0 \%)$ & $0(0 \%)$ & $1(20 \%)$ \\
\hline Female & $N$ & $0(0 \%)$ & $0(0 \%)$ & $4(26.7 \%)$ \\
\hline
\end{tabular}

Apparatus The apparatus was the same as in Experiment 1.

Stimuli We used the same set of 30 unique faces from the NimStim database as in Experiment 1. For adaptation, faces were arranged in a diamond pattern, in groups of four unique face identities conveying the same emotion, equidistant from the central fixation cross. The center of each face was $8.4^{\circ}$ from central fixation, and each face subtended $6.7^{\circ}$ visual angle in width and $7.5^{\circ}$ in height. A total of 30 possible adaptation face crowd stimuli, all displaying happy expressions, could be presented. Crowd sounds and probe morph faces were the same as in Experiment 1.

Procedure The procedure was identical to the procedure used for Experiment 1, a between-subjects design, except that groups of four faces were used as adaptation stimuli (see Fig. 4), and sound questionnaires were administered after baseline and after adaptation.

Sound questionnaire Participants were presented with $8 \mathrm{~s}$ each of positive and negative crowd sounds before adaptation and were asked to judge each set of sounds on a 7-point Likert scale, from very negative to very positive. After adaptation, participants were asked if the emotion of the sounds seemed to change over the adaptation block, and if so, they were asked to rate the emotion of the sound at the end of the block.

Data analysis The point of subjective equality was calculated as in Experiment 1. To analyze the sound questionnaire data, the mean emotional valence rating was calculated for each participant at baseline and postadaptation.

Statistical analyses Statistical analyses were performed as in Experiment 1. Paired $t$ tests were used to test for a difference in emotional rating on the emotional sound questionnaire.

\section{Results}

Out of 64 possible trials, participants completed an average of 61.09 baseline trials ( $S D=4.14$; range: $44-64)$ and an average of 62.26 postadaptation trials $(S D=3.19$; range: $49-64)$. A Kruskal-Wallis ANOVA was run to test the hypothesis that the strength of perceptual shifts varied across adaptation conditions. We found no significant main effect of adaptation condition on PSE shift, $\chi^{2}(2)=0.46, p=.80$, with a mean rank PSE shift of 34.96 for congruent, 37.05 for incongruent, and 32.90 for visual only (see Fig. 5). Although we found no significant differences in adaptation across conditions, onesample $t$ tests, using a $p$ value threshold of 0.0167 to account for multiple comparisons, indicated that the congruent and visual only, but not incongruent, conditions showed significant adaptation effects (i.e., PSE shifts were significantly different from baseline, or zero): Hc, $t(25)=-3.51, p=.002, d=$ 


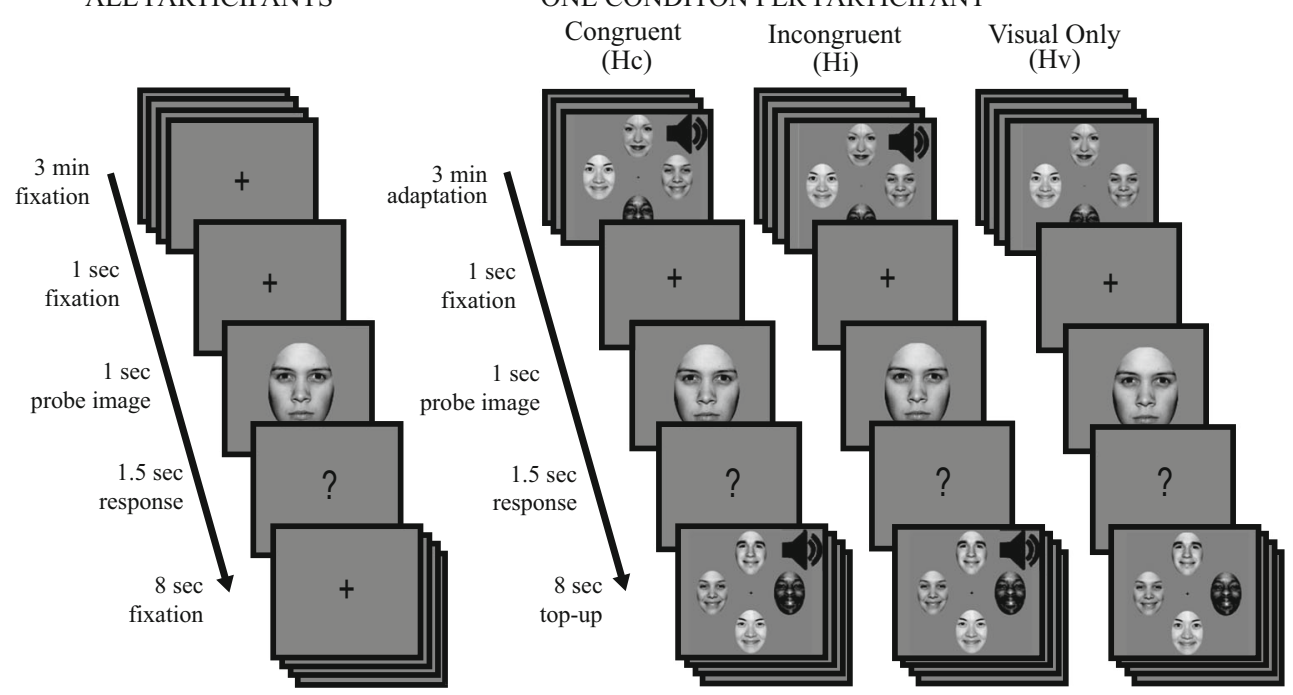

Fig. 4 Experimental procedure (Experiment 2). Participants were presented with randomly selected faces that had been morphed along an emotional continuum, from angry to happy (eight unique face identities: four males, four females). They judged each face morph as either happy or angry. For baseline, the block started with a 3-minute fixation, and participants were instructed to maintain central fixation. This was followed by a face morph presented for $1 \mathrm{~s}$, followed by a 1.5 -s response period, during which a question mark was presented and participants had to indicate whether they thought the previously presented face morph was happy or angry by pressing a key on a keyboard. Baseline consisted of 64 trials. For adaptation, participants were presented the same face morphs

and made the same judgments as during baseline. However, adaptation began with a 3-minute exposure to a $100 \%$ happy face groups, and each face judgment was followed by an 8-s top-up exposure, during which eight groups of $100 \%$ happy faces were presented, each for $1 \mathrm{~s}$. Adaptation also consisted of 64 trials. A given participant was presented with baseline followed by only one of three possible adaptation conditions: bimodal adaptation conditions, in which emotional valence was either matched (happy faces and positive sounds [Hc]), or mismatched (happy faces and negative sounds [Hi]), or a unimodal adaptation condition, in which participants were adapted to happy faces only (Hv)

$-0.69 ; \mathrm{Hv}, t(20)=-4.68, p<.001, d=-1.02 ; \mathrm{Hi}, t(21)=$ $-2.40, p=.026, d=-0.05$. Based on our a priori hypothesis (that the strength of changes in perception would be greater following congruent versus incongruent stimuli), we also tested our main comparison of interest using a Mann-Whitney $U$ test, but found no significant difference between the congruent and incongruent conditions $(p=.66)$.

We also found no significant differences in changes in slope across adaptation conditions, $F(68)=.59, p=.56$, partial $\eta^{2}=0.02$, and slope changes were not significantly different from zero $(p>.05)$ for any adaptation condition. Of note, measures quantifying perceptual changes were normalized to baseline, and we found no significant main effect of adaptation condition on perception at baseline, $\chi^{2}(2)=$ $1.78, p=.41$, with a mean rank PSE shift of 37.42 for congruent, 36.77 for incongruent, and 30.14 for visual only.

Sound questionnaire Ratings for positive and negative crowd sounds were measured at baseline and after adapt conditions, both to ensure that the sounds were emotionally salient, and to examine if participants were adapting to sounds as they were adapting to faces. On a scale from 1 to 7 , with 1 being the most negative and 7 being the most positive, the average rating of positive crowd sounds before adaptation was $6.57(S D=.60)$ and the average rating of negative crowd sounds was 1.57 ( $S D$

$=.87$ ). Paired-samples $t$ tests for positive and negative crowd sounds found significant differences between baseline and postadaptation such that sounds were rated less emotional following adaptation - that is, positive crowd sounds were rated less positive, $t(40)=4.16, p<.001$, and negative crowd sounds were rated less negative, $t(22)=-2.60, p=.016$. Importantly, despite adaptation effects, positive sounds were still rated as positive (above 4 ) and negative sounds were still rated as negative (below 4): happy pre, $t(41)=27.01, p<.001$; happy post, $t(41)=4.20, p<.001$; angry pre, $t(22)=-12.87, p$ $<.001$; angry post, $t(23)=-4.21, p<.001$ (see Figure 6).

\section{Experiment 2 discussion}

We assessed the processing of emotional crowds, with a crowd of faces presented concurrently with a crowd of voices. We hypothesized that we would see a shift in the point of subjective equality after adaptation such that (1) neutral faces would be biased to be perceived as more angry, regardless of the emotional valence of the voices, indicating that participants process groups of emotional faces in the same way as they do single faces, and (2) there would be a stronger shift in the congruent condition relative to the incongruent condition. We found that participants are indeed able to extract emotion from crowds with different identities. Shifts in the point of 
Perceptual Shift

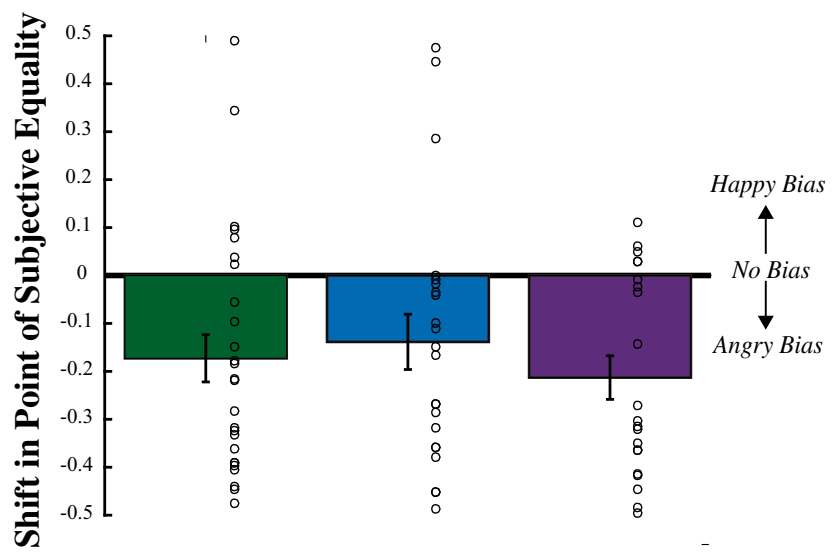

$$
\mathrm{n}=\quad \begin{array}{lll}
\mathbf{H c} & \mathbf{H i} & \mathbf{H v} \\
26 & 22 & 21
\end{array}
$$

Fig. 5 PSE shifts (Experiment 2). PSE shifts, the change in judgments of the face judged neutral at baseline, are shown for each adaptation condition. The $x$-axis depicts the adaptation condition, and the $y$-axis depicts the mean PSE shift ( \pm SEM across participants), with data from individual participants shown via open circles. A mean shift in the negative direction indicates that the unique face judged neutral at baseline for each participant looked angrier, on average, after adaptation. Conversely, a mean shift in the positive direction indicates that the unique neutral face looked happier, on average, after adaptation. After adaptation to happy faces, we observed a negative shift in all conditions, as expected: $\mathrm{Hc}, t(25)=-3.51$, $p=.002 ; \mathrm{Hv}, t(20)=-4.68, p<.001 ; \mathrm{Hi}, t(21)=-2.40, p=.026$. There were no differences in magnitude of shift between conditions, $\chi^{2}(2)=$ $0.46, p=.80$

subjective equality were in the expected direction, such that in each condition, after exposure to happy faces, participants were biased to judge neutral faces as more being angry. However, contrary to expectations, while perceptual shifts were in the expected direction, we found no difference in the strength of adaptation as a function of condition (congruent vs. incongruent). The findings from Experiment 2 replicate the findings from Experiment 1, using different visual stimuli.

Even though we found no differences in the magnitude of perceptual shift across conditions, we observed significant shifts in the expected direction. While previous work has highlighted the ability to extract featural information across groups of the same identity and over time (i.e., Haberman et al., 2009; Haberman \& Whitney, 2007), our study extends this work to show that emotional information can be extracted from a group of different identities, confirming results of previous studies (Elias, Dyer, \& Sweeny, 2017; Ji, Chen, Loeys, \& Pourtois, 2018; Li et al., 2016).

The results of Experiment 2 rule out the possibility that our failure to find differences across adaptation conditions was due to differences in numerosity between visual and auditory emotional stimuli. We also confirmed that emotional crowd sounds were consistently rated as positive for positive crowd sounds and negative for negative crowd sounds, as assessed by a survey measurement that asked participants to rate the emotion of the crowd sounds before and after adaptation. Thus, we are confident that participants were experiencing the crowd sounds as intended. Furthermore, as expected, participants adapted to the crowd sounds: subjective ratings of positive sounds were less positive and ratings of negative sounds less negative following adaptation. Of note, auditory stimuli were not captured by visual stimuli during adaptation: only three participants out of a total of 48 participants from the $\mathrm{Hc}$ and Hi conditions judged the sound as switching valence before versus after adaptation, rather than simply decreasing in valence. Finally, additional evidence that the sound was an effective stimulus is presented in the Supplemental Materials. In this additional experiment, in a new set of participants, we found that the 3-minute exposure to congruent versus incongruent sounds differentially altered ratings of negative mood (see Supplemental Materials and Supplemental Figs. S1 and

\section{Emotional Sound Rating}
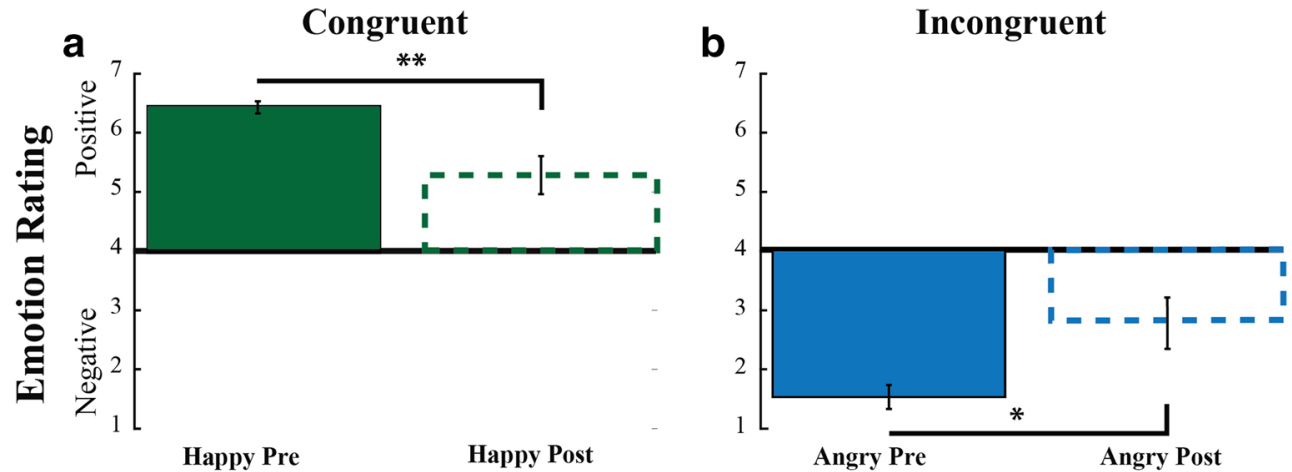

Fig. 6 Emotional sounds ratings. At baseline and postadaptation, participants rated the positive and negative crowd sounds on a scale from 1 to 7 , with 1 being the most negative and 7 being the most positive $( \pm$ SEM across participants). The asterisk represents significance at $<.05$ and double asterisk represents significance at

$<.001$. To examine how these ratings changed after adaptation, we performed paired-samples $t$ tests, and found that both sounds were judged to be significantly less emotional after adaptation: positive crowd sounds were judged less positive, $t(40)=4.16, p<.001$, and negative crowd sounds were judged less negative, $t(22)=-2.60, p=.016$ 
S2), despite the same stimuli not yielding differential perceptual aftereffects.

\section{Experiment 3}

Another factor we considered, which could explain our failure to find differences across adaptation conditions, is the possibility that participants acquired all the information they needed from visual stimuli, since faces were very salient and postadaptation judgments were only made for visual stimuli. Participants could have learned to ignore the auditory stimuli. In fact, anecdotal evidence during debriefing suggested that many participants claimed to be ignoring the sounds because they knew they must only judge the faces.

In the field of multisensory integration, the principle of inverse effectiveness states that multisensory integration is most effective when neither individual stimulus is particularly effective on its own (e.g., Stein \& Meredith, 1993; Wallace, Wilkinson, \& Stein, 1996). Thus, less salient stimuli should allow for enhanced multisensory interactions, at least up to some point as there may be a salience "sweet spot" where multisensory effects would be maximal (e.g., Ross, SaintAmour, Leavitt, Javitt, \& Foxe, 2007). While this principle has been considered in several perceptual domains (e.g., Holmes, 2009; Senkowski, Saint-Amour, Höfle, \& Foxe, 2011; Stevenson \& James, 2009), there has been limited application for the study of face processing. According to the principle of inverse effectiveness, if our face stimuli were very salient, auditory stimuli may not have interacted optimally with visual stimuli to produce a congruent advantage.

To promote multisensory interactions, we ran Experiment 3 , where we created new face stimuli, embedding our original face stimuli in white noise to decrease visual stimulus salience. Although not multisensory, previous adaptation studies within the visual modality have investigated the influence of noise on adaptation. Using a rapid double pulse adaptation design, A. Harris and Nakayama (2007) manipulated the percentage noise in which a first face stimulus was embedded to quantify subsequent adaptation effects on a second face stimulus. While increased noise decreased the neural response of the first face in noise, it did not alter subsequent adaptation of the second noiseless face in this paradigm. Interestingly, adapting to visual noise, in and of itself, has been shown to enhance subsequent processing of face stimuli (Menzel, Hayn-Leichsenring, Redies, Németh, \& Kovács, 2017). We hypothesized that decreasing the salience of the faces would help increase interactions between seen and heard emotions, resulting in stronger adaptation. We expected the strongest perceptual shifts following exposure to congruent audiovisual emotions and the weakest perceptual shifts following exposure to incongruent audiovisual emotions. Given that faces were less salient, we hypothesized that there would be no significant perceptual shift following exposure to visual stimuli alone, as previous studies have suggested that noisy visual emotional stimuli are less effective than noiseless visual emotional stimuli (Collignon et al., 2008)

\section{Method}

The method for Experiment 3 was the same as in previous experiments, with the exception of the visual stimuli presented during the adaptation period, adaptation faces, being embedded in Gaussian distributed white noise using Adobe Photoshop.

Participants An additional 75 participants, between the ages of 18 and 50, were recruited from the University of Massachusetts Boston community participated. We aimed to collect roughly the same number of participants as in Experiment 1. Ten participants were excluded: three due to biased behavioral responses, two due to experimenter error, four due to insufficient trial numbers, and one due to incompletion of the study. Our final sample included 65 participants, 50 females (mean age $=22.00$ years; $S D=5.26$; range: $18-50$ years), and 13 males (mean age $=25.08$ years; $S D=6.44$; range: $19-38$ years). Two participants did not provide their age or gender (please see Table 3 for a detailed breakdown of demographic information for our final sample).

Table 3 Demographic information (Experiment 3)

\begin{tabular}{|c|c|c|c|c|}
\hline $\begin{array}{l}\text { Demographics } \\
\text { Mean age } \\
(S D)\end{array}$ & & $\begin{array}{l}\text { Congruent } \\
24.25 \\
(7.68)\end{array}$ & $\begin{array}{l}\text { Incongruent } \\
21.95 \\
(4.29)\end{array}$ & $\begin{array}{l}\text { Visual } \\
21.81 \\
(4.29)\end{array}$ \\
\hline \multicolumn{5}{|l|}{ White } \\
\hline Male & $N$ & $4(66.7 \%)$ & $1(20 \%)$ & $1(50 \%)$ \\
\hline Female & $N$ & $6(42.9 \%)$ & $7(41.2 \%)$ & $7(36.8 \%)$ \\
\hline \multicolumn{5}{|l|}{ Hispanic } \\
\hline Male & $N$ & $0(0 \%)$ & $2(40 \%)$ & $0(0 \%)$ \\
\hline Female & $N$ & $2(14.3 \%)$ & $5(29.4 \%)$ & $3(15.8 \%)$ \\
\hline \multicolumn{5}{|c|}{ African/African American } \\
\hline Male & $N$ & $1(16.7 \%)$ & $1(20 \%)$ & $0(0 \%)$ \\
\hline Female & $N$ & $2(14.3 \%)$ & $0(0 \%)$ & $3(15.8 \%)$ \\
\hline \multicolumn{5}{|l|}{ Asian } \\
\hline Male & $N$ & $0(0 \%)$ & $1(20 \%)$ & $1(50 \%)$ \\
\hline Female & $N$ & $2(14.3 \%)$ & $4(23.5 \%)$ & $3(15.8 \%)$ \\
\hline \multicolumn{5}{|l|}{ Multiracial } \\
\hline Male & $N$ & $1(16.7 \%)$ & $0(0 \%)$ & $0(0 \%)$ \\
\hline Female & $N$ & $1(7.1 \%)$ & $1(5.9 \%)$ & $1(5.3 \%)$ \\
\hline \multicolumn{5}{|l|}{ Unspecified } \\
\hline Male & $N$ & $0(0 \%)$ & $0(0 \%)$ & $0(0 \%)$ \\
\hline Female & $N$ & $1(7.1 \%)$ & $0(0 \%)$ & $3(15.8 \%)$ \\
\hline
\end{tabular}


Participants reported normal hearing and normal or corrected-to-normal vision, and provided written informed consent. Compensation was $\$ 12 / \mathrm{hr}$ or extra credit for an eligible undergraduate course. All experimental procedures and protocols were approved by the University of Massachusetts Boston Institutional Review Board and complied with the Declaration of Helsinki.

Apparatus The apparatus was the same as in Experiments 1 and 2 .

Stimuli We used the same set of 30 faces from the NimStim database as in Experiment 1. Crowd sounds and probe face morphs were the same as those used in Experiment 1. The one change was that the $100 \%$ happy faces presented during initial adaptation and top-up adaptation were embedded in Gaussiandistributed white noise, and contrast was reduced by $50 \%$ (see Fig. 7).

Procedure The procedure was the same as the procedure for the previous experiments, a between-subjects design.

Data analysis The point of subjective equality was calculated as in Experiments 1 and 2. As before, since all adapting faces were happy, we expected participants exposed to congruent or incongruent stimuli to show a negative bias following adaptation. Since adapting faces were now embedded in white noise and were less salient than the unedited faces used in Experiments 1 and 2, we expected participants to have (1) the strongest negative bias following adaptation to congruent responses and (2) the weakest negative bias following adaptation to incongruent stimuli. We had no clear expectation for how biases following adaptation to only visual stimuli (faces embedded in noise) would compare to our congruent and incongruent conditions.

Statistical analyses Statistical analysis was the same as in Experiments 1 and 2.

\section{Results}

Out of 64 possible trials, participants completed an average of 60.80 baseline trials $(S D=4.59$; range: $41-64)$ and an average of 61.60 postadaptation trials $(S D=5.97$; range: $35-64)$. A one-way ANOVA was run to test the hypothesis that the strength of changes in perception varied across adaptation conditions. We expected the shift in PSE to be greater for the congruent compared with the incongruent condition. Because the faces are embedded in visual noise, we do not expect the visual-only condition to produce a significant

\section{Baseline Condition ALL PARTICIPANTS}

Adaptation Condition ONE CONDITON PER PARTICIPANT

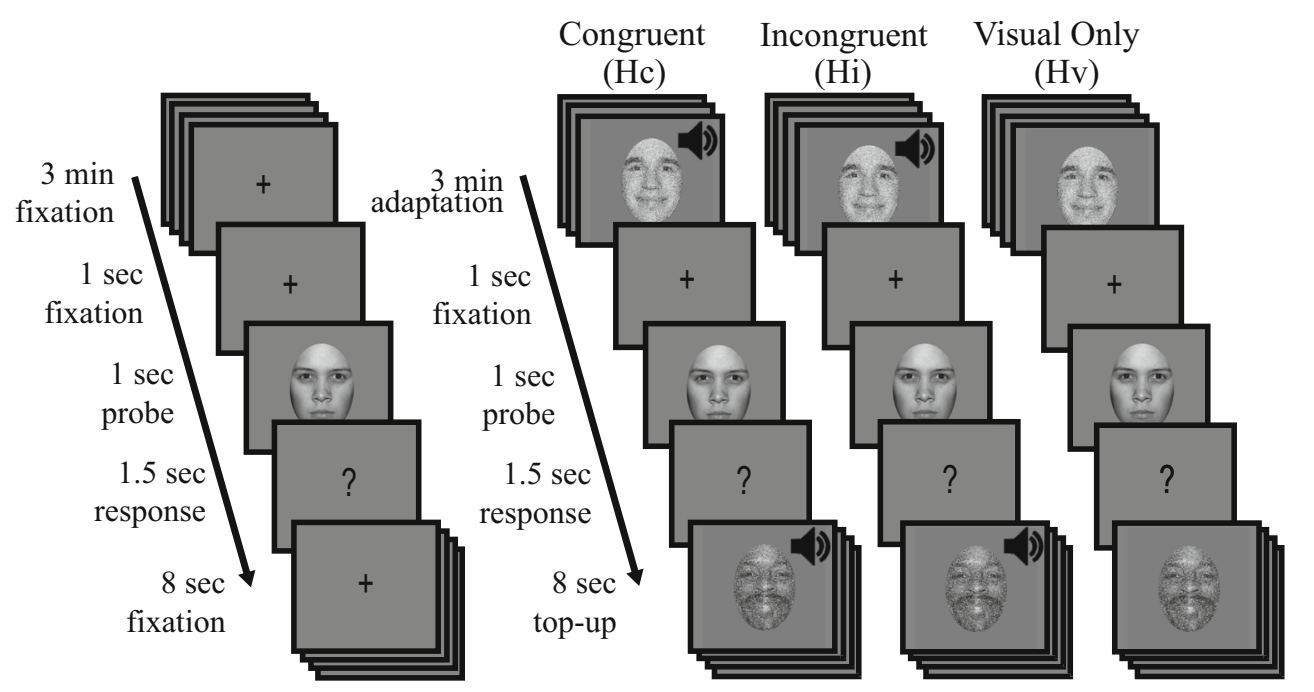

Fig. 7 Experimental procedure (Experiment 3). Participants were presented randomly selected faces that had been morphed along an emotional continuum, from angry to happy (eight unique face identities: four males, four females). They judged each face morph as either happy or angry. For baseline, the block started with a 3-minute fixation, and participants were instructed to maintain central fixation. This was followed by a face morph presented for $1 \mathrm{~s}$, followed by a 1.5 -s response period, during which a question mark was presented, and participants had to indicate whether they thought the previously presented face morph was happy or angry by pressing a key on a keyboard. Baseline consisted of 64 trials. For adaptation, participants were presented the same face morphs

and made the same judgments as during baseline. However, adaptation began with a 3-minute exposure to $100 \%$ happy faces embedded in white noise, and each face judgment was followed by an 8-s top-up exposure, during which eight $100 \%$ emotional faces, again embedded in noise, were presented, each for $1 \mathrm{~s}$. Adaptation also consisted of 64 trials. A given participant was presented with baseline followed by only one of three possible adaptation conditions: bimodal adaptation conditions, in which emotional valence was either matched (happy faces and positive sounds $[\mathrm{Hc}]$ ), or mismatched (happy faces and negative sounds [Hi]), or a unimodal adaptation condition, in which participants were adapted to happy faces only (Hv) 
aftereffect, and had no clear prediction of how it would compare to the congruent or incongruent condition. We found no significant main effect of adaptation condition on PSE shift, $F(2,64)=1.678, p>.05$, partial $\eta^{2}=0.051$ (see Fig. 8). Onesample $t$ tests indicated that congruent and incongruent conditions showed significant adaptation effects, with PSE shifts significantly different from baseline, or zero: $\mathrm{Hc}, t(20)=$ $-4.97, p<.001, d=-1.08$; Hi, $t(21)=-2.63, p=.008, d=$ -0.56 . Because we had an a priori hypothesis that the perceptual shift would be greater for the congruent relative to the incongruent condition, we also performed an independentsamples $t$ test to test for differences in the magnitude of PSE shifts between the congruent and incongruent conditions. We observed a trend for a larger PSE shift following exposure to congruent versus incongruent stimuli, $t(41)=-1.84, p=.073$, $d=0.56$.

For additional measures we considered, we found no significant differences in changes in slope between conditions, $F(2,64)=.533, p>.05$, partial $\eta^{2}=0.017$, and slope changes were not significantly different from zero for any condition $(p \mathrm{~s}$ $>.05$ ). Furthermore, we found no significant main effect of adaptation condition on perception at baseline, $\chi^{2}(2)=2.34, p$ $=.31$, with a mean rank PSE shift of 35.24 for congruent, 35.86 for incongruent, and 28.00 for visual only).

While we did not have an explicit prediction for the magnitude of an aftereffect resulting from adapting to faces embedded in noise, we imagined that the aftereffect would be

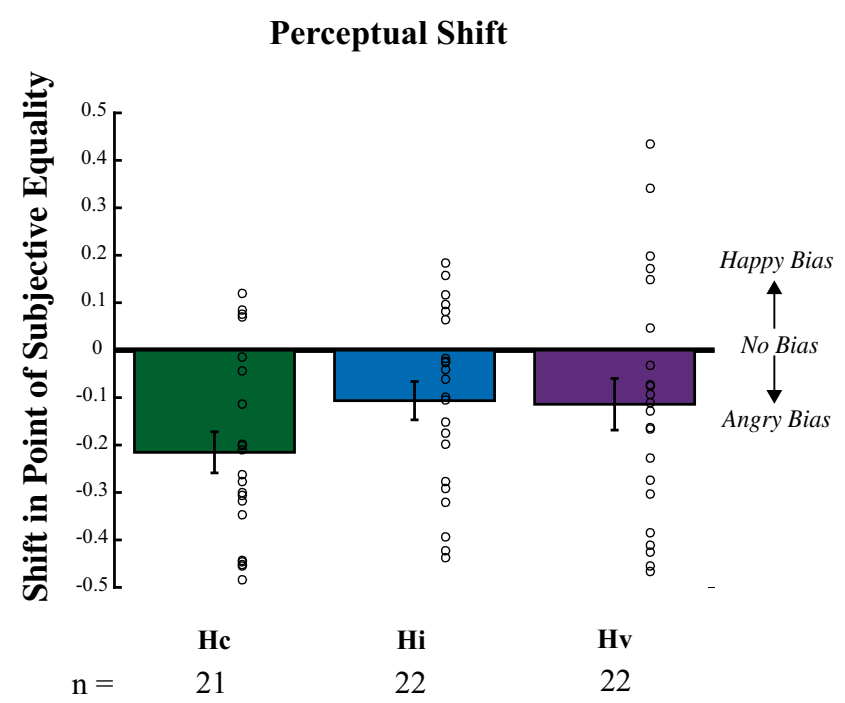

Fig. 8 PSE shifts (Experiment 3). PSE shifts, the change in judgments of the face judged neutral at baseline, are shown for each adaptation condition. The $x$-axis depicts the adaptation condition, and the $y$-axis depicts the mean PSE shift ( \pm SEM across participants), with data from individual participants shown via open circles. A mean shift in the negative direction indicates that the unique face judged neutral at baseline for each participant looked angrier, on average, after adaptation. Conversely, a mean shift in the positive direction indicates that the unique neutral face looked happier, on average, after adaptation. There was a trend for a larger shift following exposure to congruent relative to incongruent emotional stimuli, $t(41)=-1.84, p=.073$ smaller than what was elicited by fully salient faces. To assess this, we compared the visual only conditions from Experiment 1 (fully salient faces) and Experiment 3 (less salient faces embedded in noise). We observed a significant difference with a significant decrease in the perceptual shift found in Experiment 3, with faces of reduced salience, relative to Experiment 1, with fully salient faces, $t(38)=-2.69, p=$ $.011, d=0.86$ (see Fig. 9).

\section{Experiment 3 discussion}

In Experiment 3, adaptation faces were embedded in white noise to decrease salience. Given the theory of inverse effectiveness, we expected a significant difference in the magnitude of PSE shift between congruent and incongruent adaptation, despite finding no significant difference between the congruent and incongruent conditions in Experiments 1 or 2. We found a trend for a greater PSE shift after adaptation to congruent versus incongruent happy emotions. This is in agreement with a related study (Collignon et al., 2008), one of the few studies using emotional faces embedded in noise. This

\section{Visual Only Comparison}

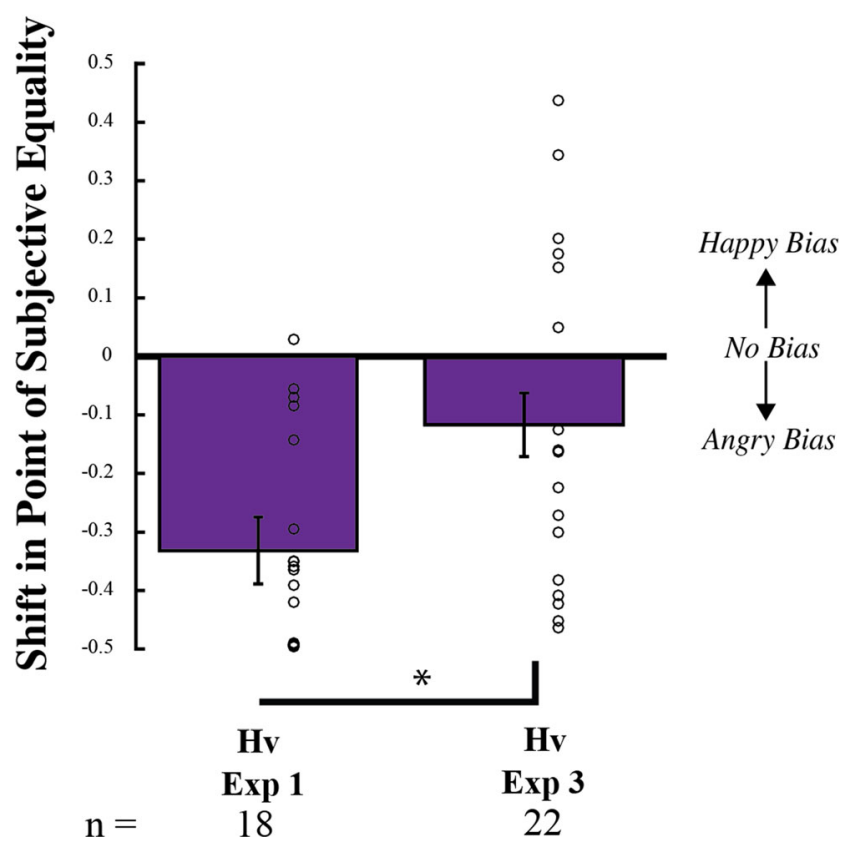

Fig. 9 Visual only, Hv, adaptation condition across Experiments 1 and 3. PSE shifts, the change in judgments of the face judged neutral at baseline are shown for the visual only (Hv) adaptation conditions of Experiment 1 (fully salient faces) and Experiment 3 (faces of reduced salience). The $x$ axis depicts the adaptation condition, and the $y$-axis depicts the mean PSE shift ( $\pm S E M$ across participants), with data from individual participants shown via open circles. The asterisk represents significance of $p<.05$. A mean shift in the negative direction indicates that the unique face judged neutral at baseline for each participant looked angrier, on average, after adaptation. There was a significantly larger shift to judge faces more negatively following adaptation to fully salient happy faces relative to less salient happy faces, $t(38)=-2.69, p=.011$ 
study assessed congruent, incongruent, and visual only fearful and disgusted faces and voices. Unlike our study, this study did not use an adaptation paradigm, the emotions tested were different and both negative, the face stimuli were dynamic rather than static, and participants attended to the faces or to the voices. Despite these differences, this related study found an advantage in reaction time for congruent over incongruent audiovisual emotions using faces of decreased salience (Collignon et al., 2008).

\section{Experiment 4}

A major limitation of our previous experiments is that attention is not controlled for. While Experiment 3 showed promising results for enhanced multisensory integration using faces in noise instead of fully salient faces, in Experiment 4 we sought to enhance adaptation effects by controlling for attention.

Previous experiments have shown that attention enhances adaptation in several domains, including motion perception (e.g., Rezec, Krekelberg, \& Dobkins, 2004), spatial frequency perception (e.g., Carrasco, Loula, \& Ho, 2006), and even face perception (e.g., Rhodes et al., 2011). While there is not yet much research on how attention enhances the processing of emotions in faces per se, we hypothesized that directing attention to the emotion of the face would enhance adaptation, optimize multisensory integration, and highlight the difference in perceptual aftereffects between congruent and incongruent adaptation conditions. In the last experiment of this series, we controlled for attention by including catch trials during adaptation periods, directing participants to pay attention to the emotion of the $100 \%$ happy faces presented throughout adaptation.

\section{Method}

Participants A total of 43 additional participants, between the ages of 18 and 49 years, were recruited from the UMass Boston community. Three participants were excluded due to experimenter error. Our final sample included 40 participants -29 females (mean age $=23.86$ years; $S D=6.17$; range: $18-47$ years), and nine males (mean age $=26.56$ years; $S D=8.60$; range: $18-43$ years). One participant did not report their sex, and one was intersex (please see Table 4 for detailed demographic information for this final sample).

Participants reported normal hearing and normal or corrected-to-normal vision, and provided written informed consent. Participants were compensated with either $\$ 12 / \mathrm{hr}$ or extra credit for approved psychology courses. All experimental procedures and protocols were approved by the University of Massachusetts Boston Institutional Review Board and complied with the Declaration of Helsinki.
Table 4 Demographic information (Experiment 4)

\begin{tabular}{|c|c|c|c|}
\hline $\begin{array}{l}\text { Demographics } \\
\text { Mean age }(S D)\end{array}$ & & $\begin{array}{l}\text { Congruent } \\
23.45(6.72)\end{array}$ & $\begin{array}{l}\text { Incongruent } \\
25.84(6.72)\end{array}$ \\
\hline \multicolumn{4}{|l|}{ White } \\
\hline Male & $N$ & $2(50 \%)$ & $1(20 \%)$ \\
\hline Female & $N$ & $6(37.5 \%)$ & $6(46.2 \%)$ \\
\hline \multicolumn{4}{|l|}{ Hispanic } \\
\hline Male & $N$ & $0(0 \%)$ & $0(0 \%)$ \\
\hline Female & $N$ & $3(18.8 \%)$ & $3(23.1 \%)$ \\
\hline \multicolumn{4}{|c|}{ African/African American } \\
\hline Male & $N$ & $0(0 \%)$ & $0(0 \%)$ \\
\hline Female & $N$ & $1(6.3 \%)$ & $1(7.7 \%)$ \\
\hline \multicolumn{4}{|l|}{ Asian } \\
\hline Male & $N$ & $1(25 \%)$ & $3(60 \%)$ \\
\hline Female & $N$ & $5(31.3 \%)$ & $0(0 \%)$ \\
\hline \multicolumn{4}{|l|}{ Multiracial } \\
\hline Male & $N$ & $0(0 \%)$ & $1(20 \%)$ \\
\hline Female & $N$ & $0(0 \%)$ & $0(0 \%)$ \\
\hline \multicolumn{4}{|l|}{ Unspecified } \\
\hline Male & $N$ & $1(25 \%)$ & $0(0 \%)$ \\
\hline Female & $N$ & $1(6.3 \%)$ & $3(23.1 \%)$ \\
\hline
\end{tabular}

Apparatus Participants were seated in a dark soundproof chamber. Visual stimuli were presented on a Tobii TX300 screen and auditory stimuli were presented via speakers on either side of the screen. Participants were seated $60 \mathrm{~cm}$ from the screen.

Stimuli Sound stimuli and adaptation faces and probe faces were the same as in Experiment 3, embedded in noise. Catch trial images are $10 \%, 40 \%$, and $80 \%$ happy images (instead of $20 \%, 40 \%$, and $80 \%$ ).

Procedure The experiment began with three practice trials, similarly to the previous experiments. Participants were instructed to maintain central fixation throughout the experiment, but eye gaze was not monitored. Following practice trials, each participant completed one baseline and one adapt condition (see Fig. 10). Given the longer duration of initial adaptation and top-up adaptation due to the addition of catch trials (described below), participants completed 24 total trials for baseline as well as 24 total trials after adaptation.

The same procedures were followed for baseline as described for Experiment 3, except that we only tested congruent and incongruent conditions. Furthermore, adaptation was similar to previous procedures, with the notable addition of 22 catch trials interspersed randomly throughout the $180 \mathrm{~s}$ initial adaptation to control for attention, and the addition of one catch trial during each $8 \mathrm{~s}$ top-up adaptation. Participants were asked to judge the catch trial face as happy or angry. 


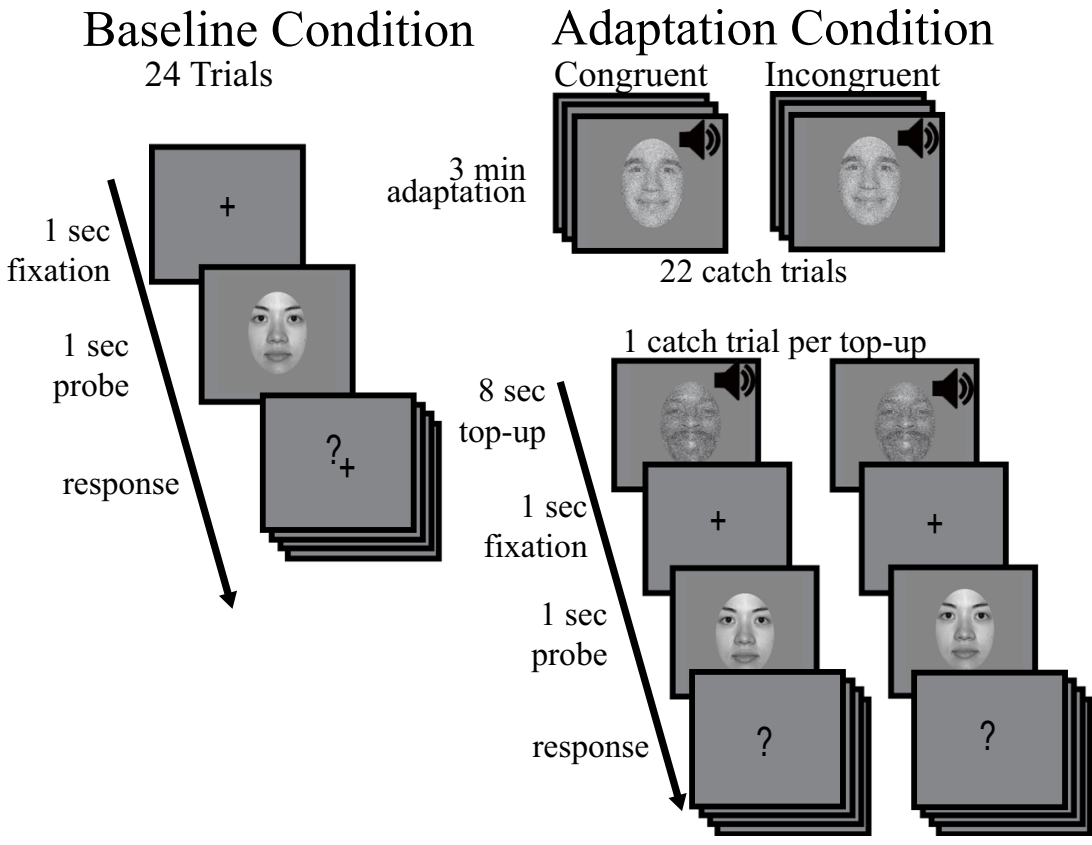

Fig. 10 Experimental procedure (Experiment 4). Participants were presented randomly selected faces that had been morphed along an emotional continuum, from angry to happy (four unique face identities: two males, two females). They judged each face morph as either happy or angry. The baseline block began with a fixation cross presented for $1 \mathrm{~s}$. This was followed by a face morph presented for $1 \mathrm{~s}$, followed by a response period up to $6 \mathrm{~s}$, during which a question mark was presented, and participants had to indicate whether they thought the previously presented face morph was happy or angry by pressing a key on a keyboard. Baseline consisted of 24 trials. For adaptation, participants were presented the same face morphs and made the same judgments as during baseline.

Data analysis The point of subjective equality was calculated as in Experiments 1-3. As before, since all adapting faces were happy, we expected participants exposed to congruent or incongruent stimuli to show a negative bias following adaptation. Since catch trials were added to enhance attention towards the emotion of the face stimuli, we again expected participants to show more negative bias following adaptation to congruent versus incongruent audiovisual stimuli.

Statistical analyses Planned statistical analyses include an independent-samples $t$ test to test the hypothesis that adaptation would be stronger following exposure to congruent versus incongruent emotional stimuli.

\section{Results}

Out of 24 possible trials, participants completed an average of 23.85 baseline trials $(S D=.43$; range: $22-24)$ and an average of 23.88 adaptation trials $(S D=.65$; range: $20-24)$. Since data were normally distributed, an independent-samples $t$ test was run to test the hypothesis that the strength of adaptation differed between the two conditions. We found a significant difference, such that PSE shifts were significantly larger for
However, adaptation began with a 3 -minute exposure to $100 \%$ happy faces embedded in white noise, and each face judgment was followed by an 8 -s top-up exposure, during which eight $100 \%$ emotional faces, again embedded in noise, were presented, each for $1 \mathrm{~s}$. Adaptation also consisted of 24 trials. A given participant was presented with baseline, followed by only one of the two possible adaptation conditions: emotional valence was either matched (happy faces and positive sounds [Hc]), or mismatched (happy faces and negative sounds [Hi]). Catch trials, asking participants to judge the emotion or gender of a face, were interspersed randomly during initial adaptation, and once within each top-up.

congruent versus incongruent adaptation conditions, $t(38)=$ $-2.43, p=.020, d=-0.77$ (see Fig. 11).

One-sample $t$ tests indicated that PSE shift for the congruent conditions was significantly different from baseline, or zero, but for incongruent was not: Hc, $t(19)=-7.73, p<$ $.001, d=-2.44 ; \mathrm{Hi}, t(19)=-1.89, p=.07, d=-.60$. We found no significant differences in changes in slope between conditions, $t(19)=.18, p=.13, d=.06$. While we found a significant increase in slope for the incongruent condition: $\mathrm{Hi}$, $t(19)=3.34, p<.05, d=1.10$, the congruent condition showed no significant change in slope: Hc, $t(19)=1.28, p=.24, d=$ .34. Of note, measures quantifying perceptual changes were normalized to baseline, and we found no significant main effect of adaptation condition on perception at baseline, $t(38)$ $=.85, p=.40$.

\section{Discussion}

In Experiment 4, we controlled attention during adaptation. Catch trials during initial adaptation and top-up adaptation were used to direct attention to the face since participants had to judge faces as happy or angry. Directing attention to face emotion enhanced multisensory effects during 


\section{Perceptual Shift}

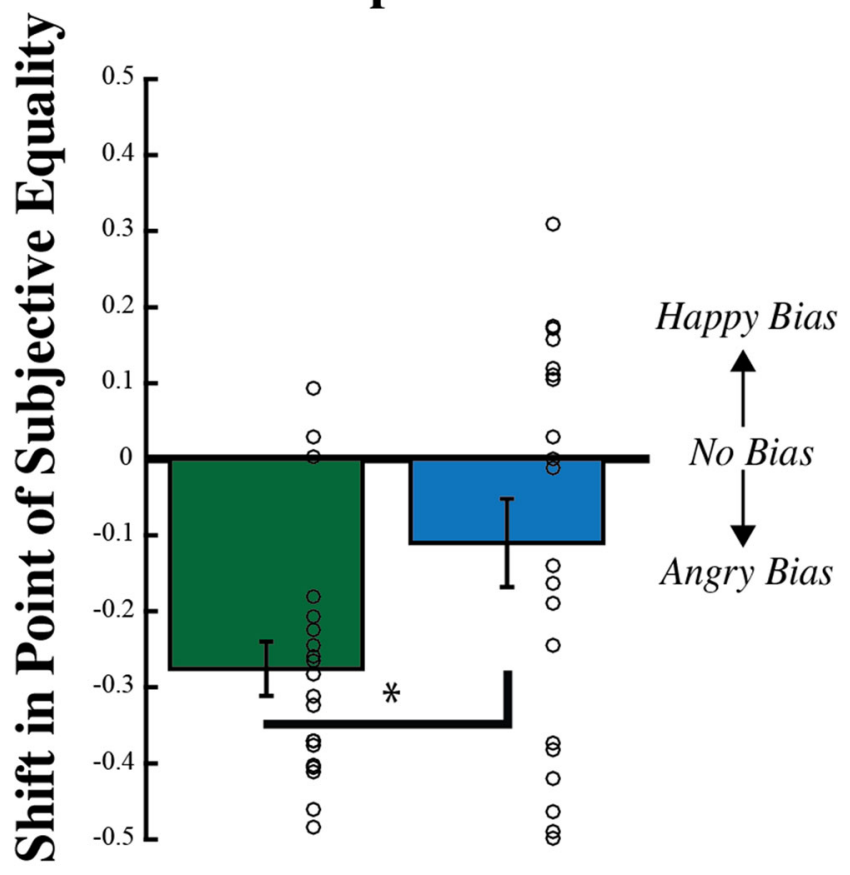

$\mathrm{Hc}$

Hi

\section{$\mathbf{n}=\mathbf{2 0} \quad \mathbf{2 0}$}

Fig. 11 PSE shifts (Experiment 5). PSE shifts, the change in judgments of the face judged neutral at baseline, are shown for each adaptation condition. The $x$-axis depicts the adaptation condition and the $y$-axis depicts the mean PSE shift ( $\pm S E M$ across participants), with data from individual participants shown via open circles. The asterisk represents significance of $<.05$. A mean shift in the negative direction indicates that the unique face judged neutral at baseline for each participant looked angrier, on average, after adaptation. Conversely, a mean shift in the positive direction indicates that the unique neutral face looked happier, on average, after adaptation. After adaptation to happy faces, we observed a negative shift in the congruent and incongruent conditions: Hc, $t(19)=-7.73, p<.001, d=-2.44$; Hi, $t(19)=-1.89, p$ $=.04, d=-.60$. There was a significantly larger shift following exposure to congruent stimuli relative to incongruent stimuli, $t(38)=-2.43, p=$ $.020, d=-0.77$

adaptation. For the same faces embedded in noise as in Experiment 3 the trend we observed of congruent adaptation effects being stronger than incongruent adaptation, was enhanced in Experiment 4 when stimuli had to be attended to. These results suggest that directing attention towards the emotion of the face during adaptation strengthened the difference in perceptual aftereffects between congruent and incongruent conditions.

\section{General discussion}

Across a series of four experiments, we assessed the relative influence of auditory emotional informational on the processing of visual emotional information, quantified via the strength of adaptation (a PSE shift) serving as a proxy for the strength of the visual representation. Previous work has yielded mixed results as to whether or not congruent audiovisual emotional stimuli could provide a processing advantage over incongruent audiovisual stimuli. Unlike previous adaptation studies using unique face-voice pairings for only a few individual face identities (e.g., Little et al., 2013; Wang et al., 2016), we used a wide range of facial identities (30 unique identities) and unassociated crowd sounds to assess emotional processing. In each of our four perceptual experiments, adaptation to happy faces yielded a negative shift in the PSE, a contrastive perceptual aftereffect. But, only in our last experiment, in which the visual salience of emotional faces was decreased and attention was directed to the emotion of the face during adaptation, did we observe the expected results (significantly stronger adaptation, a greater PSE shift) for congruent versus incongruent audiovisual emotional stimuli.

We hypothesized that adaptation to faces in noise $(\mathrm{Hv})$ would induce weaker aftereffects compared with adaptation to the fully salient faces used in Experiment 1, and observed a significantly larger perceptual aftereffect in condition $\mathrm{Hv}$ following adaptation to fully salient noiseless (Experiment 1) compared with noisy faces of reduced salience (Experiment 4). This weakened adaptation effect confirms that embedding the faces in noise does in fact reduce stimulus salience, and consequently visual adaptation efficacy in our experimental paradigm. This decreased visual salience contributes to enhancing multisensory interactions, enhancing perceptual shifts for congruent relative to incongruent emotional conditions. Such an effect is predicted by the principle of inverse effectiveness, which suggests that highly salient stimuli are not optimized for crossmodal integration or interaction and that suboptimal stimuli yield stronger crossmodal interactions. Given that previous studies using different multisensory stimuli find a sweet spot of stimulus salience, where the benefits for multisensory interaction are optimal (e.g., Chow et al., 2020; Ross et al., 2007), it is possible that the congruency effects we observed for multisensory emotional stimuli could have been better optimized by finding the sweet spot of salience for visual faces by considering crossmodal effects under differing noise levels. Importantly, it was not until attention was controlled for, in Experiment 4, that we observed a significant difference between congruent and incongruent conditions. This suggests that, in our task, decreased salience alone may not be sufficient to optimize multisensory interactions; attention towards the emotional stimuli during adaptation might also be needed. Our findings accord with some of the results of Collignon et al. (2008), who used faces embedded in noise and found enhanced emotional processing for congruent compared with incongruent audiovisual emotional stimuli. The study by Collignon and colleagues did not use adaptation and focused on the negative emotions of fear and disgust; but of note, although they directed participants to 
attend to visual or auditory emotional stimuli, the faces themselves were intrinsically more attention grabbing as they were dynamic faces, unlike the static faces used in our study.

While, a review is beyond the scope of this paper, more recently work has begun to consider behavioral as well as neural correlates of crossmodal emotional processing (for review, see Jessen \& Kotz, 2013; Klasen, Kreifelts, Chen, Seubert, \& Mathiak, 2014). Although some ERP studies find enhanced behavioral and neuronal processing for congruent bimodal compared with unimodal emotional stimuli (Chen et al., 2016), other studies find neuronal but not behavioral enhancement (DelleVigne, Kornreich, Verbanck, \& Campanella, 2014; Kokinous, Kotz, Tavano, \& Schröger, 2015). This suggests differences between unimodal and bimodal processing may not always cross the threshold to influence behavior, even when present at the neuronal level. Furthermore, differences in results across studies may arise due to differences in stimulus salience and the allocation of attention to visual or auditory stimuli.

\section{Limitations}

It is possible that other variables, not accounted for here, could have minimized the perceptual shifts we were trying to quantify. In our study, we asked participants to rate the emotion of the positive and negative crowd sounds, both to validate that sounds were perceived as positive or negative, not neutral, and to determine if the subjective experience of the sounds changed after adaptation. We confirmed that participants rated the positive sounds positively and negative sounds negatively, and found that both positive and negative emotional sounds became significantly less emotional after adaptation. This suggests an auditory adaptation effect occurring concurrently with the visual adaptation effect. Importantly, the sounds were nonetheless perceived to be emotional and judged as being the expected emotional valence. There is a possibility that crowd sounds could be less effective towards the end of the adaptation block just as visual faces could be less effective towards the end of the adaptation block. Further studies will need to examine this effect in a more systematic way.

Another limitation is that all our experiments were conducted using a between-subjects design, whereas a withinsubjects design would provide more power and might better account for individual differences. We ran these experiments using a between-subjects design to avoid adaptation effects from one condition influencing subsequent conditions, as we found in pilot studies. Such confounds are especially prevalent in the long-duration ( 3 minutes) adaptation paradigm used here, which were run within a single day of testing. In cases where a within-subjects design has been used for adaptation studies within a single testing day, such as the study by Wang et al. (2016), a short-duration (4 s) adaptation paradigm was used. Furthermore, use of a between-subjects design allows for easier translation of such studies to developmental populations, which we are pursuing.
Overall, although we did control for attention in Experiment 4, there is a much broader debate regarding attentional load and emotional processing, which was not addressed here. While the classical view has been that emotion is processed regardless of availability of attentional resources (e.g., Vuilleumier, Armony, Driver, \& Dolan, 2001), recent evidence has challenged this view, asserting that participants may not process emotion while engaged in a concurrent attentionally demanding task (e.g., Pessoa, 2005; Pessoa, McKenna, Gutierrez, \& Ungerleider, 2002). Whereas the former theory suggests that emotional information is immune to the constraints of attentional resources, the latter suggests that emotional information is subject to the same limitations as other types of information processing. Future studies will need to better assess the role of attention in the processing of unimodal and bimodal emotional stimuli. Attentional influences in bimodal audiovisual conditions are especially important to consider, as a given sound might not be equally engaging or distracting across participants, and the relative dominance of visual and auditory processing may vary (e.g., Shinn-Cunningham, 2017; Sörqvist \& Rönnberg, 2014).

The four experiments reported here measure perceptual changes, across roughly 240 participants, all demonstrating contrastive perceptual aftereffects, but suggesting that emotional information from multiple sources is only beneficial under certain circumstances. Namely, a congruent processing advantage seems to manifest best when visual emotional stimuli are reduced in salience, and attended to. Complementary effects might be found in the auditory modality, if auditory emotions were embedded in noise and attended. Our results align with the complementary "cocktail party effect": if you are trying to understand what your friend is saying at a noisy cocktail party, you may better understand what you hear if you use not only your ears but also your eyes (e.g., Cherry, 1953). This arises because we can process speech information more accurately if we use correlated visual cues to aid in reading lips while listening to what is being said than if we use either visual or auditory speech information alone (e.g., Besle, Fort, Delpuech, \& Giard, 2004; van Wassenhove, Grant, \& Poeppel, 2005). If the speech sounds are fully salient and clear, in and of themselves, we may disregard visual cues, as they may not further assist in the processing of speech information. This process is fast and automatic, being hard to ignore (e.g., McGurk \& MacDonald, 1976). According to our results from Experiment 4, congruent emotional information can provide a perceptual advantage over incongruent, or conflicting, emotional information. Follow-up studies are needed to determine if there is a sweet spot for emotional salience that maximizes emotional interactions across the senses and to better understand the role of crossmodal attention in the processing of emotion.

Acknowledgements We thank a wonderful and dedicated team of undergraduate research assistants, especially Daniel Harris, Ryan McCarty, Alexia Williams, Anh Phan, Brandon Mui, Abrar Ahmed, Julio Verne, Kaitlin Parent, Valerie, Goutama, and Erinda Morina for their help with 
data collection and analysis. And finally, much of this work would not have been possible without generous support from the UMass Boston Dean's Research Fund and the UMass Boston Department of Psychology Research Fund (V.M.C.) and the UMass Boston undergraduate RES fund (V.G., K.P., J.V., A.C.). All authors declare that they have no conflict of interest and that this work has not been previously published.

Open practices statement The data and materials from all experiments reported in this manuscript will be made available upon email request to the corresponding author and will be uploaded to the Open Science Framework. None of the experiments reported was preregistered.

\section{References}

Alvarez, G. A. (2011). Representing multiple objects as an ensemble enhances visual cognition. Trends in Cognitive Sciences, 15(3), 122-131. doi:https://doi.org/10.1016/j.tics.2011.01.003

Ariely, D. (2001). Seeing sets: Representation by statistical properties. Psychological Science, 12(2), 157-162.

Barth, H., Kanwisher, N., \& Spelke, E. (2003). The construction of large number representations in adults. Cognition, 86(3), 201-221. doi: https://doi.org/10.1016/S0010-0277(02)00178-6

Besle, J., Fort, A., Delpuech, C., \& Giard, M.-H. (2004). Bimodal speech: Early suppressive visual effects in human auditory cortex. European Journal of Neuroscience, 20(8), 2225-2234. doi:https://doi.org/10. 1111/j.1460-9568.2004.03670.x

Bestelmeyer, P., Maurage, P., Rouger, J., Latinus, M., \& Belin, P. (2014). Adaptation to Vocal Expressions Reveals Multistep Perception of Auditory Emotion. Journal of Neuroscience, 34(24), 8098-8105. doi:https://doi.org/10.1523/JNEUROSCI.4820-13.2014

Bestelmeyer, P. E. G., Rouger, J., DeBruine, L. M., \& Belin, P. (2010). Auditory adaptation in vocal affect perception. Cognition, 117(2), 217-223. doi:https://doi.org/10.1016/j.cognition.2010.08.008

Brady, T. F., \& Alvarez, G. A. (2011). Hierarchical encoding in visual working memory: Ensemble statistics bias memory for individual items. Psychological Science, 22(3), 384-392. doi:https://doi.org/ 10.1177/0956797610397956

Brainard, D. H. (1997). The Psychophysics Toolbox. Spatial Vision, 10(4), 433-436. https://doi.org/10.1163/156856897X00357

Carrasco, M., Loula, F., \& Ho, Y.-X. (2006). How attention enhances spatial resolution: Evidence from selective adaptation to spatial frequency. Perception \& Psychophysics, 68(6), 1004-1012. doi: https://doi.org/10.3758/BF03193361

Chen, X., Pan, Z., Wang, P., Yang, X., Liu, P., You, X., \& Yuan, J. (2016). The integration of facial and vocal cues during emotional change perception: EEG markers. Social Cognitive and Affective Neuroscience, 11(7), 1152-1161. doi:https://doi.org/10.1093/scan/ nsv083

Cherry, C. (1953). The cocktail party effect. The Journal of the Acoustical Society of America, 25(5), 975-979.

Chong, S. C., \& Treisman, A. (2003). Representation of statistical properties. Vision Research, 43(4), 393-404. doi:https://doi.org/10. 1016/S0042-6989(02)00596-5

Chow, H. M., Leviyah, X., \& Ciaramitaro, V. M. (2020). Individual differences in multisensory interactions: The influence of temporal phase coherence and auditory salience on visual contrast sensitivity. Vision, 4(1), 12

Collignon, O., Girard, S., Gosselin, F., Roy, S., Saint-Amour, D., Lassonde, M., \& Lepore, F. (2008). Audio-visual integration of emotion expression. Brain Research, 1242, 126-135. doi:https:// doi.org/10.1016/j.brainres.2008.04.023

de Fockert, J., \& Wolfenstein, C. (2009). Short article: Rapid extraction of mean identity from sets of faces. Quarterly Journal of Experimental Psychology, 62(9), 1716-1722. doi:https://doi.org/10.1080/ 17470210902811249

de Gelder, B., \& Vroomen, J. (2000). The perception of emotions by ear and by eye. Cognition \& Emotion, 14(3), 289-311. doi:https://doi. org/10.1080/026999300378824

Delle-Vigne, D., Kornreich, C., Verbanck, P., \& Campanella, S. (2014). Subclinical alexithymia modulates early audio-visual perceptive and attentional event-related potentials. Frontiers in Human Neuroscience, 8. doi:https://doi.org/10.3389/fnhum.2014.00106

Elias, E., Dyer, M., \& Sweeny, T. D. (2017). Ensemble Perception of Dynamic Emotional Groups. Psychological Science, 28(2), 193203. doi:https://doi.org/10.1177/0956797616678188

Faul, F., Erdfelder, E., Lang, A.-G., \& Buchner, A. (2007). G*Power 3: A flexible statistical power analysis program for the social, behavioral, and biomedical sciences. Behavior Research Methods, 39(2), 175191. doi:https://doi.org/10.3758/BF03193146

Flom, R., \& Bahrick, L. E. (2007). The development of infant discrimination of affect in multimodal and unimodal stimulation: The role of intersensory redundancy. Developmental Psychology, 43(1), 238252. doi:https://doi.org/10.1037/0012-1649.43.1.238

Fox, C. J., \& Barton, J. J. S. (2007). What is adapted in face adaptation? The neural representations of expression in the human visual system. Brain Research, 1127, 80-89. doi:https://doi.org/10.1016/j. brainres.2006.09.104

Gallup, A. C., Hale, J. J., Sumpter, D. J. T., Garnier, S., Kacelnik, A., Krebs, J. R., \& Couzin, I. D. (2012). Visual attention and the acquisition of information in human crowds. Proceedings of the National Academy of Sciences of the United States of America, 109(19), 7245-7250. doi:https://doi.org/10.1073/pnas.1116141109

Grossmann, T., Striano, T., \& Friederici, A. D. (2006). Crossmodal integration of emotional information from face and voice in the infant brain. Developmental Science, 9(3), 309-315. doi:https://doi.org/10. 1111/j.1467-7687.2006.00494.x

Haberman, J., Harp, T., \& Whitney, D. (2009). Averaging facial expression over time. Journal of Vision, 9(11), 1-1. doi:https://doi.org/10. $1167 / 9.11 .1$

Haberman, J., \& Whitney, D. (2007). Rapid extraction of mean emotion and gender from sets of faces. Current Biology, 17(17), R751R753. doi:https://doi.org/10.1016/j.cub.2007.06.039

Harris, A., \& Nakayama, K. (2007). rapid face-selective adaptation of an early extrastriate component in MEG. Cerebral Cortex, 17(1), 63 70. doi:https://doi.org/10.1093/cercor/bhj124

Harris, A., \& Nakayama, K. (2008). Rapid Adaptation of the M170 Response: Importance of Face Parts. Cerebral Cortex, 18(2), 467476. doi:https://doi.org/10.1093/cercor/bhm078

Harris, D. A., \& Ciaramitaro, V. M. (2016). Interdependent Mechanisms for Processing Gender and Emotion: The Special Status of Angry Male Faces. Frontiers in Psychology, 7. doi:https://doi.org/10.3389/ fpsyg.2016.01046

Herz, R. S., McCall, C., \& Cahill, L. (1999). Hemispheric lateralization in the processing of odor pleasantness versus odor names. Chemical Senses, 24(6), 691-695. doi:https://doi.org/10.1093/chemse/24.6. 691

Holmes, N. P. (2009). Inverse effectiveness, multisensory integration, and the bodily self: Some statistical considerations. Consciousness and Cognition, 18(3), 762-765. doi:https://doi.org/10.1016/j. concog.2009.04.009

Hsu, S.-M., \& Young, A. (2004). Adaptation effects in facial expression recognition. Visual Cognition, 11(7), 871-899. doi:https://doi.org/ 10.1080/13506280444000030

Izen, S. C., Lapp, H. E., Harris, D. A., Hunter, R. G., \& Ciaramitaro, V. M. (2019). Seeing a face in a crowd of emotional voices: Changes in 
perception and cortisol in response to emotional information across the senses. Brain Sciences, 9(8), 176. doi:https://doi.org/10.3390/ brainsci9080176

Jessen, S., \& Kotz, S. A. E. (2013). On the role of crossmodal prediction in audiovisual emotion perception. Frontiers in Human Neuroscience, 7. doi:https://doi.org/10.3389/fnhum.2013.00369

Ji, L., Chen, W., Loeys, T., \& Pourtois, G. (2018). Ensemble representation for multiple facial expressions: Evidence for a capacity limited perceptual process. Journal of Vision, 18(3), 17-17. doi:https://doi. org $/ 10.1167 / 18.3 .17$

Jordan, K. E., \& Brannon, E. M. (2006). The multisensory representation of number in infancy. Proceedings of the National Academy of Sciences of the United States of America, 103(9), 3486-3489. doi: https://doi.org/10.1073/pnas.0508107103

Klasen, M., Kreifelts, B., Chen, Y.-H., Seubert, J., \& Mathiak, K. (2014). Neural processing of emotion in multimodal settings. Frontiers in Human Neuroscience, 8. doi:https://doi.org/10.3389/fnhum.2014. 00822

Kleiner M, Brainard D, Pelli D, 2007, "What's new in Psychtoolbox-3?" Perception 36 ECVP Abstract Supplement.

Kobayashi, T., Hiraki, K., \& Hasegawa, T. (2005). Auditory-visual intermodal matching of small numerosities in 6-month-old infants. Developmental Science, 8(5), 409-419. doi:https://doi.org/10. 1111/j.1467-7687.2005.00429.x

Kokinous, J., Kotz, S. A., Tavano, A., \& Schröger, E. (2015). The role of emotion in dynamic audiovisual integration of faces and voices. Social Cognitive and Affective Neuroscience, 10(5), 713-720. doi: https://doi.org/10.1093/scan/nsu105

Li, H., Ji, L., Tong, K., Ren, N., Chen, W., Liu, C. H., \& Fu, X. (2016). Processing of individual items during ensemble coding of facial expressions. Frontiers in Psychology, 7. doi:https://doi.org/10. 3389/fpsyg.2016.01332

Little, A. C., Feinberg, D. R., DeBruine, L. M., \& Jones, B. C. (2013). Adaptation to faces and voices: Unimodal, cross-modal, and sexspecific effects. Psychological Science, 24(11), 2297-2305. doi: https://doi.org/10.1177/0956797613493293

McGurk, H., \& MacDonald, J. (1976). Hearing lips and seeing voices. Nature, 264(5588), 746-748.

Menzel, C., Hayn-Leichsenring, G. U., Redies, C., Németh, K., \& Kovács, G. (2017). When noise is beneficial for sensory encoding: Noise adaptation can improve face processing. Brain and Cognition, 117, 73-83. doi:https://doi.org/10.1016/j.bandc.2017.06.006

Müller, V. I., Habel, U., Derntl, B., Schneider, F., Zilles, K., Turetsky, B. I., \& Eickhoff, S. B. (2011). Incongruence effects in crossmodal emotional integration. NeuroImage, 54(3), 2257-2266. doi:https:// doi.org/10.1016/j.neuroimage.2010.10.047

Ng, M., Ciaramitaro, V. M., Anstis, S., Boynton, G. M., \& Fine, I. (2006). Selectivity for the configural cues that identify the gender, ethnicity, and identity of faces in human cortex. Proceedings of the National Academy of Sciences of the United States of America, 103(51), 19552-19557. doi:https://doi.org/10.1073/pnas.0605358104

Niedenthal, P. M. (2007). Embodying emotion. Science, 316(5827), 1002-1005. doi:https://doi.org/10.1126/science.1136930

Parkes, L., Lund, J., Angelucci, A., Solomon, J. A., \& Morgan, M. (2001). Compulsory averaging of crowded orientation signals in human vision. Nature Neuroscience, 4(7), 739-744.

Pelli, D. G. (1997). The VideoToolbox software for visual psychophysics: Transforming numbers into movies. Spatial Vision 10:437-442.

Pessoa, L. (2005). To what extent are emotional visual stimuli processed without attention and awareness? Current Opinion in Neurobiology, 15(2), 188-196. doi:https://doi.org/10.1016/j.conb.2005.03.002

Pessoa, L., McKenna, M., Gutierrez, E., \& Ungerleider, L. G. (2002). Neural processing of emotional faces requires attention. Proceedings of the National Academy of Sciences, 99(17), 1145811463.
Pye, A., \& Bestelmeyer, P. E. G. (2015). Evidence for a supra-modal representation of emotion from cross-modal adaptation. Cognition, 134, 245-251. doi:https://doi.org/10.1016/j.cognition.2014.11.001

Rezec, A., Krekelberg, B., \& Dobkins, K. R. (2004). Attention enhances adaptability: Evidence from motion adaptation experiments. Vision Research, 44(26), 3035-3044. doi:https://doi.org/10.1016/j.visres. 2004.07.020

Rhodes, G., Jeffery, L., Evangelista, E., Ewing, L., Peters, M., \& Taylor, L. (2011). Enhanced attention amplifies face adaptation. Vision Research, 51(16), 1811-1819. doi:https://doi.org/10.1016/j.visres. 2011.06.008

Robins, D. L., Hunyadi, E., \& Schultz, R. T. (2009). Superior temporal activation in response to dynamic audio-visual emotional cues. Brain and Cognition, 69(2), 269-278. doi:https://doi.org/10.1016/ j.bandc.2008.08.007

Roesch, E. B., Sander, D., Mumenthaler, C., Kerzel, D., \& Scherer, K. R. (2010). Psychophysics of emotion: The QUEST for emotional attention. Journal of Vision, 10(3), 4-4. doi:https://doi.org/10.1167/ 10.3.4

Ross, L. A., Saint-Amour, D., Leavitt, V. M., Javitt, D. C., \& Foxe, J. J. (2007). Do you see what I am saying? Exploring Visual enhancement of speech comprehension in noisy environments. Cerebral Cortex, 17(5), 1147-1153. doi:https://doi.org/10.1093/cercor/ bhl024

Rutherford, M. D., Chattha, H. M., \& Krysko, K. M. (2008). The use of aftereffects in the study of relationships among emotion categories, 34(1), 27-40. doi:https://doi.org/10.1037/0096-1523.34.1.27

Senkowski, D., Saint-Amour, D., Höfle, M., \& Foxe, J. J. (2011). Multisensory interactions in early evoked brain activity follow the principle of inverse effectiveness. NeuroImage, 56(4), 2200-2208. doi:https://doi.org/10.1016/j.neuroimage.2011.03.075

Shinn-Cunningham, B. (2017). Cortical and sensory causes of individual differences in selective attention ability among listeners with normal hearing thresholds. Journal of Speech, Language, and Hearing Research: JSLHR, 60(10), 2976-2988. doi:https://doi.org/10.1044/ 2017 JSLHR-H-17-0080

Skuk, V. G., \& Schweinberger, S. R. (2013). Adaptation aftereffects in vocal emotion perception elicited by expressive faces and voices. PLOS ONE, 8(11), e81691. doi:https://doi.org/10.1371/journal. pone. 0081691

Sörqvist, P., \& Rönnberg, J. (2014). Individual differences in distractibility: An update and a model. PsyCh Journal, 3(1), 42-57. doi:https:// doi.org/10.1002/pchj.47

Starkey, P., Spelke, E. S., \& Gelman, R. (1990). Numerical abstraction by human infants. Cognition, 36(2), 97-127. doi:https://doi.org/10. 1016/0010-0277(90)90001-Z

Stein, B. E., \& Meredith, M. A. (1993). The merging of the senses. Cambridge, MA: MIT Press.

Stevenson, R. A., \& James, T. W. (2009). Audiovisual integration in human superior temporal sulcus: Inverse effectiveness and the neural processing of speech and object recognition. NeuroImage, 44(3), 1210-1223. doi:https://doi.org/10.1016/j.neuroimage.2008.09.034

Sweeny, T. D., \& Whitney, D. (2014). Perceiving crowd attention: Ensemble perception of a crowd's gaze. Psychological Science, 25(10), 1903-1913.

Tottenham, N., Tanaka, J. W., Leon, A. C., McCarry, T., Nurse, M., Hare, T. A., ... Nelson, C. (2009). The NimStim set of facial expressions: Judgments from untrained research participants. Psychiatry Research, 168(3), 242-249. doi:https://doi.org/10.1016/j.psychres. 2008.05.006

van Wassenhove, V., Grant, K. W., \& Poeppel, D. (2005). Visual speech speeds up the neural processing of auditory speech. Proceedings of the National Academy of Sciences of the United States of America, 102(4), 1181-1186. doi:https://doi.org/10.1073/pnas.0408949102

Vogel, M., Monesson, A., \& Scott, L. S. (2012). Building biases in infancy: The influence of race on face and voice emotion matching. 
Developmental Science, 15(3), 359-372. doi:https://doi.org/10. 1111/j.1467-7687.2012.01138.x

Vuilleumier, P., Armony, J. L., Driver, J., \& Dolan, R. J. (2001). Effects of attention and emotion on face processing in the human brain. Neuron, 30(3), 829-841. doi:https://doi.org/10.1016/S08966273(01)00328-2

Walker, D., \& Vul, E. (2014). Hierarchical encoding makes individuals in a group seem more attractive. Psychological Science, 25(1), 230235. doi:https://doi.org/10.1177/0956797613497969

Wallace, M. T., Wilkinson, L. K., \& Stein, B. E. (1996). Representation and integration of multiple sensory inputs in primate superior colliculus. Journal of Neurophysiology, 76(2), 1246-1266. doi: https://doi.org/10.1152/jn.1996.76.2.1246

Wang, X., Guo, X., Chen, L., Liu, Y., Goldberg, M. E., \& Xu, H. (2016). Auditory to Visual Cross-Modal Adaptation for Emotion: Psychophysical and Neural Correlates. Cerebral Cortex, bhv321. doi:https://doi.org/10.1093/cercor/bhv321

Webster, M. A., Kaping, D., Mizokami, Y., \& Duhamel, P. (2004). Adaptation to natural facial categories. Nature, 428(6982), 557561. doi:https://doi.org/10.1038/nature02420
Webster, M. A., \& MacLeod, D. I. A. (2011). Visual adaptation and face perception. Philosophical Transactions of the Royal Society B: Biological Sciences, 366(1571), 1702-1725. doi:https://doi.org/10. 1098/rstb.2010.0360

Wichmann, F. A., \& Hill, N. J. (2001). The psychometric function: I. Fitting, sampling, and goodness of fit. Perception \& Psychophysics, 63(8), 1293-1313. doi:https://doi.org/10.3758/ BF03194544

Zald, D. H., \& Pardo, J. V. (1997). Emotion, olfaction, and the human amygdala: Amygdala activation during aversive olfactory stimulation. Proceedings of the National Academy of Sciences of the United States of America, 94(8), 4119-4124. doi:https://doi.org/10.1073/ pnas.94.8.4119

Publisher's note Springer Nature remains neutral with regard to jurisdictional claims in published maps and institutional affiliations. 